\title{
Stable pulse solutions for the nonlinear Schrödinger equation with higher order dispersion management
}

\author{
Jamison T. Moeser, Christopher K.R.T. Jones, Vadim Zharnitsky
}

September 11, 2003

\begin{abstract}
The evolution of optical pulses in fiber optic communication systems with strong, higher order dispersion management is modeled by a cubic nonlinear Schrödinger equation with periodically varying linear dispersion at second and third order. Through an averaging procedure, we derive an approximate model for the slow evolution of such pulses, and show that this system possesses a stable ground state solution. Furthermore, we characterize the ground state numerically. The results explain the experimental observation of higher order DM solitons, providing theoretical justification for modern communication systems design.
\end{abstract}

\section{Introduction}

\subsection{Conventional dispersion management}

The technique of dispersion management (DM), introduced in the early eighties [16] and refined during the past decade [27], has emerged as the dominant technology for high bandwidth data transmission through optical fibers. In a dispersion managed fiber link, short sections of fiber with opposite linear dispersion characteristics are joined together in a periodically repeated structure, forming a fiber whose linear dispersion is effectively canceled out over each period of dispersion management. In such a system, the characteristic length of local dispersion is much shorter than that of nonlinearity or average dispersion, so that on the scale of a typical dispersion management segment, the effects of nonlinearity and average dispersion can be made small relative to those of the local dispersion. In this regime, destabilizing effects such as four-wave mixing $[2,5,22]$ and Gordon-Haus jitter $[14,36]$ are minimized.

In the case of dispersion management at second order, the propagation equation for the wave envelope can be written in the dimensionless form

$$
i u_{z}+d_{2}(z) u_{t t}+\epsilon|u|^{2} u=0
$$


with the dispersion coefficient $d_{2}(z)$ decomposed into its varying and average components

$$
d_{2}(z)=\tilde{d}_{2}(z)+\epsilon \alpha_{2}
$$

where $\int_{0}^{1} \tilde{d}_{2}\left(z^{\prime}\right) d z^{\prime}=0$. Typically $d_{2}(z)$ is piecewise constant and periodic, and the parameter $\epsilon$ corresponds to the ratio of the characteristic length scales of local dispersion to that of nonlinearity and average dispersion [1, 10].

The system performance of dispersion managed fiber links is truly remarkable $[8,25]$. Not only are stable pulse structures observed for $\alpha_{2}>0$, the focusing (anomalous) regime for NLS [9], but also for the case when $\alpha_{2} \leq 0$ $[14,28]$. These DM solitons are characterized by nearly Gaussian central peaks and rapidly decaying secondary peaks which comprise the tails [20, 28]. Also, in contrast to the soliton solutions for the NLS equation, the DM solitons possess a nontrivial quadratic phase component, namely chirp [10].

The energy of the DM soliton is higher than that of the corresponding NLS soliton with the same full width at half maximum (FWHM) and average dispersion $[29,37]$. This makes DM solitons more resistant to the effects of spontaneously emitted amplifier noise, giving dispersion managed systems a higher signal to noise ratio than traditional soliton based systems [34]. Also, this energy enhancement can be exploited to reduce energy variation per channel in wavelength division multiplexing (WDM) systems operating near zero average dispersion [29].

The first analytical results for DM solitons were obtained in the late nineties, when an averaged equation describing the slow evolution of solutions to (1) was derived in [10] through path averaging and in [1] through multiple scales expansion. A rigorous justification for this averaged equation was given in [38], where, moreover, it was shown that for $\alpha_{2}>0$, the Hamiltonian corresponding to the averaged equation possesses a ground state solution in the class of functions $\mathcal{A}_{\lambda}=\left\{u: \int_{\mathbb{R}}|u|^{2}=\lambda, \int_{\mathbb{R}}\left|u_{t}\right|^{2}<\infty\right\}$. These results indicate the existence of a stable, stationary solution to the averaged equation that propagates nearly periodically for (1) on time scales up to $\mathcal{O}\left(\frac{1}{\epsilon}\right)$. The existence of a standing wave solution for the averaged equation in this regime was also established in [12] by means of a general theorem on bifurcation of solutions from the essential spectrum. Furthermore, the existence of a ground state for the case $\alpha_{2}=0$ was recently demonstrated [13].

\subsection{Higher order dispersion management}

Waves of the form $\exp (i D(\omega) z-i \omega t)$ traveling through an optical fiber satisfy the dispersion relation

$$
D(\omega)=\frac{n(\omega) \omega}{c},
$$

where $D(\omega)$ is termed the propagation constant, $n(\omega)$ is the index of refraction, $\omega$ is the frequency, and $c$ is the speed of light in a vacuum [3]. Thus, in general, the propagation constant is a complicated function of frequency. Explicit 
forumlas for $D(\omega)$ are generally unknown, and in the derivation of the evolution equation for the electric field's slowly varying amplitude, $D(\omega)$ is approximated by its Taylor polynomial in a neighborhood of the carrier frequency.

$$
D(\omega) \simeq d_{0}+d_{1}\left(\omega-\omega_{0}\right)+d_{2}\left(\omega-\omega_{0}\right)^{2}+\ldots
$$

where $d_{n}=\frac{D^{(n)}\left(\omega_{0}\right)}{n !}$. In the derivation of the conventional dispersion management model (1), one assumes that pulses are sufficiently narrow in the frequency domain, $\left|\omega-\omega_{0}\right|<<1$, so that $D(\omega)$ is accurately approximated by its quadratic Taylor polynomial, and the effects of changes in the values of $d_{2}$ in a neighborhood of $\omega_{0}$ are neglected. However, for the propagation of pulses which are broader in the frequency domain, the inclusion of the cubic term in the Taylor approximation is necessary, with the resulting model taking into account variations in $d_{2}$.

This additional term in the Taylor series gives rise to a third order linear dispersive term in the governing NLS-type equation for the electric field's slowly varying envelope [3]. In single channel systems, third order dispersion generally causes an asymmetric broadening of pulses. Moreover, in WDM systems, which utilize many optical channels separated in the frequency domain, third order dispersion can prevent conventional dispersion compensation across neighboring channels.

A natural way to surmount these difficulties is to manage dispersion at both second and third order. By utilizing this technique of higher order dispersion management (HODM), the asymmetric broadening that takes place for ultrashort optical pulses in single channel systems with conventional DM is almost exactly compensated for. Furthermore, in WDM systems, HODM makes it possible to compensate for dispersion over many neighboring frequency channels simultaneously. In fact, advances in fiber manufacturing techniques [18] have made it possible to incorporate this idea into new optical fibers, termed dispersion slope compensating fibers, and recent experiments have yielded impressive results $[7,11,19,23,26]$.

The evolution of optical pulses in a fiber with dispersion management at second and third order is governed by the following dimensionless nonlinear Schrödinger type equation [24]

$$
i u_{z}+d_{2}(z) u_{t t}+i d_{3}(z) u_{t t t}+\epsilon_{n l}|u|^{2} u=0
$$

with

$$
d_{j}(z)=\tilde{d}_{j}(z)+\epsilon_{j} \alpha_{j}
$$

and

$$
\int_{0}^{1} \tilde{d}_{j}\left(z^{\prime}\right) d z^{\prime}=0
$$


Here the $\alpha_{j}, j=2,3$ are order one measures of average dispersion at second and third order, respectively, $\epsilon_{n l}$ is a small parameter representing the ratio of characteristic lengths of the local dispersions to the nonlinearity, and the $\epsilon_{j}$ are small parameters representing the ratio of characteristic lengths of the local dispersions to the average dispersions. The dispersion coefficients $d_{j}(z)$, $j=2,3$, are piecewise constant and, due to the manufacture process, periodic with the same period, here normalized to be 1 . In the operating regimes we consider, the parameters satisfy $\epsilon_{3}<<\epsilon_{n l} \sim \epsilon_{2}$, so we set $\epsilon=\epsilon_{n l}=\epsilon_{2}$, neglect the effects of average third order dispersion by setting $\alpha_{3}=0$, and consider the equation

$$
\begin{array}{r}
i u_{z}+d_{2}(z) u_{t t}+i d_{3}(z) u_{t t t}+\epsilon|u|^{2} u=0 \\
d_{2}(z)=\tilde{d}_{2}(z)+\epsilon \alpha_{2} \\
d_{3}(z)=\tilde{d}_{3}(z) .
\end{array}
$$

We develop an averaging theory for (2), and show that for the case $\alpha_{2}>0$, the corresponding averaged equation possesses a ground state solution which propagates nearly periodically for the full equation. Furthermore, we solve the Euler-Lagrange equation numerically, revealing the structure of this new DM soliton. We also report that analysis for the case $\alpha_{2}=0$ will appear elsewhere.

\section{Averaging}

Solutions of (2) evolve on two distinct spatial scales, which suggests performing an averaging procedure. We note that the analysis in this section does not require the condition $\alpha_{3}=0$, but is necessary later when proving the existence of ground states .

\subsection{Averaged equation}

We first perform the transformation $u(z, t)=\mathcal{L}(z)\{v(z, t)\}$, where $\mathcal{L}\{\cdot\}$ is the unitary semigroup for the linear evolution equation

$$
i u_{z}+\tilde{d}_{2}(z) u_{t t}+i \tilde{d}_{3}(z) u_{t t t}=0 .
$$

The operator is easily computed via Fourier transform

$$
\mathcal{L}(z)\{v(0, t)\}=\frac{1}{\sqrt{2 \pi}} \int_{\mathbb{R}} \theta(z, k) \hat{v}(0, k) \exp (i k t) d k
$$

where

$$
\theta(z, k)=\exp \int_{0}^{z}-i\left[k^{2} \tilde{d}_{2}(\tau)-k^{3} \tilde{d}_{3}(\tau)\right] d \tau .
$$

We observe that $\mathcal{L}(z)$ is an isometry on $H^{s}(\mathbb{R})$ for all $s \in \mathbb{R}$. Moreover, due to the periodicity of $\tilde{d}_{2}(z)$ and $\tilde{d}_{3}(z)$, both $\theta(z, k)$ and $\mathcal{L}(z)$ are periodic in $z$. For 
ease of notation, we henceforth suppress the variable dependencies of $v$.

Using

$\frac{\partial u}{\partial z}=\frac{\partial(\mathcal{L}(z)\{v\})}{\partial z}=\mathcal{L}(z)\left\{\frac{\partial v}{\partial z}\right\}+i \tilde{d}_{2}(z) \frac{\partial^{2}(\mathcal{L}(z)\{v\})}{\partial t^{2}}-\tilde{d}_{3}(z) \frac{\partial^{3}(\mathcal{L}(z)\{v\})}{\partial t^{3}}$

we obtain by direct substitution into (2), the evolution equation for $v$

$$
i \frac{\partial v}{\partial z}+\epsilon\left(\alpha_{2} \frac{\partial^{2} v}{\partial t^{2}}+C(z)\{v\}\right)=0
$$

where

$$
C(z)\{v\}=\mathcal{L}(-z)\left\{|\mathcal{L}(z)\{v\}|^{2} \mathcal{L}(z)\{v\}\right\} .
$$

Formally the averaged equation is

$$
i \frac{\partial v}{\partial z}+\epsilon\left(\alpha_{2} \frac{\partial^{2} v}{\partial t^{2}}+\langle C\rangle\{v\}\right)=0
$$

where

$$
\langle C\rangle\{v\}=\int_{0}^{1} \mathcal{L}\left(-z^{\prime}\right)\left\{\left|\mathcal{L}\left(z^{\prime}\right)\{v\}\right|^{2} \mathcal{L}\left(z^{\prime}\right)\{v\}\right\} d z^{\prime}
$$

In Fourier space, (7) takes the form

$$
i \frac{\partial \hat{v}}{\partial z}+\epsilon\left(-k^{2} \alpha_{2} \hat{v}+\langle\hat{C}\rangle\{v\}\right)=0
$$

with

$\langle\hat{C}\rangle\{v\}=\int_{0}^{1} \int_{\mathbb{R}^{3}} \delta\left(k-k_{1}+k_{2}-k_{3}\right) \Theta\left(z^{\prime}, k, k_{1}, k_{2}, k_{3}\right) \hat{v}_{1}(z) \overline{\hat{v}}_{2}(z) \hat{v}_{3}(z) d k_{1} d k_{2} d k_{3} d z^{\prime}$.

Here $\hat{v}_{i}(z)=\hat{v}\left(z, k_{i}\right)$ and

$\Theta\left(z^{\prime}, k, k_{1}, k_{2}, k_{3}\right)=\exp \int_{0}^{z^{\prime}} i\left\{-\tilde{d}_{2}(\tau)\left[k_{1}^{2}-k_{2}^{2}+k_{3}^{2}-k^{2}\right]+\tilde{d}_{3}(\tau)\left[k_{1}^{3}-k_{2}^{3}+k_{3}^{3}-k^{3}\right]\right\} d \tau$.

Performing the integration over $z^{\prime}$ in (10) gives

$$
\langle\hat{C}\rangle\{v\}=\int_{\mathbb{R}^{3}} \delta\left(k-k_{1}+k_{2}-k_{3}\right) \Theta_{l}\left(k, k_{1}, k_{2}, k_{3}\right) \hat{v}_{1}(z) \overline{\hat{v}}_{2}(z) \hat{v}_{3}(z) d k_{1} d k_{2} d k_{3}
$$

where

$$
\Theta_{l}\left(k, k_{1}, k_{2}, k_{3}\right)=\int_{0}^{1} \Theta\left(z^{\prime}, k, k_{1}, k_{2}, k_{3}\right) d z^{\prime}
$$


is a bounded function on $\mathbb{R}^{4}$. The averaged equation (7) corresponds to the variational equation

$$
u_{z}=J \nabla\langle H\rangle
$$

where $J=-i$ is a skew-symmetric operator, $\nabla$ is the Fréchet derivative, and $\langle H\rangle$ is the Hamiltonian

$$
\langle H\rangle(v)=\alpha_{2} \int_{\mathbb{R}}\left|v_{t}\right|^{2} d t-\frac{1}{2} \int_{0}^{1} \int_{\mathbb{R}}\left|\mathcal{L}\left(z^{\prime}\right)\{v\}\right|^{4} d t d z^{\prime}
$$

We note that $\langle H\rangle(v)$ is a bounded functional on $H^{1}(\mathbb{R})$, as

$\|\mathcal{L}\{v\}\|_{L^{4}}^{4} \leq M\left\|\frac{\partial(\mathcal{L}\{v\})}{\partial t}\right\|_{L^{2}} \cdot\|\mathcal{L}\{v\}\|_{L^{2}}^{3}=M\left\|\mathcal{L}\left\{v_{t}\right\}\right\|_{L^{2}} \cdot\|\mathcal{L}\{v\}\|_{L^{2}}^{3}=M\left\|v_{t}\right\|_{L^{2}} \cdot\|v\|_{L^{2}}^{3}$

where we have used the Gagliardo-Nirenberg inequality [4] and the fact that $\mathcal{L}(z)$ is an isometry on any space $H^{s}(\mathbb{R})$.

We comment briefly on the regularity of the averaged operator $\langle C\rangle$. We will first show that $\langle C\rangle\{\cdot\}$ is bounded on $H^{s}(\mathbb{R})$ for $s>\frac{1}{2}$. Now

$$
\begin{array}{r}
|\langle\hat{C}\rangle\{v\}|=\left|\int_{\mathbb{R}^{3}} \delta\left(k-k_{1}+k_{2}-k_{3}\right) \Theta_{l}\left(k, k_{1}, k_{2}, k_{3}\right) \hat{v}\left(k_{1}\right) \overline{\hat{v}}\left(k_{2}\right) \hat{v}\left(k_{3}\right) d k_{1} d k_{2} d k_{3}\right| \\
\leq\left\|\Theta_{l}\right\|_{L^{\infty}\left(\mathbb{R}^{4}\right)} \int_{\mathbb{R}^{3}} \delta\left(k-k_{1}+k_{2}-k_{3}\right)\left|\hat{v}\left(k_{1}\right) \overline{\hat{v}}\left(k_{2}\right) \hat{v}\left(k_{3}\right)\right| d k_{1} d k_{2} d k_{3} \\
\leq\left\|\Theta_{l}\right\|_{L^{\infty}\left(\mathbb{R}^{4}\right)} \int_{\mathbb{R}^{2}}\left|\hat{v}\left(k_{1}\right)\right|\left|\hat{\hat{v}}\left(k_{2}\right) \| \hat{v}\left(k-k_{1}+k_{2}\right)\right| d k_{1} d k_{2}
\end{array}
$$

If we denote $\hat{\mathcal{I}}\{v\}=\int_{\mathbb{R}^{2}}\left|\hat{v}\left(k_{1}\right)\right|\left|\overline{\hat{v}}\left(k_{2}\right)\right|\left|\hat{v}\left(k-k_{1}+k_{2}\right)\right| d k_{1} d k_{2}$, then by the above estimate, it suffices to show that

$$
\|\mathcal{I}\{v\}\|_{H^{s}(\mathbb{R})} \leq\|v\|_{H^{s}(\mathbb{R})}^{3}
$$

Now for any $u$ and $w \in H^{s}(\mathbb{R})$, we have that

$$
\|u w\|_{H^{s}(\mathbb{R})} \leq\|u\|_{H^{s}(\mathbb{R})}\|w\|_{H^{s}(\mathbb{R})},
$$

or equivalently in Fourier domain,

$$
\|\hat{u} * \hat{w}\|_{L_{w}^{2}(\mathbb{R})} \leq\|\hat{u}\|_{L_{w}^{2}(\mathbb{R})}\|\hat{w}\|_{L_{w}^{2}(\mathbb{R})},
$$

where $*$ is the convolution operator and

$$
\|u\|_{L_{w}^{2}(\mathbb{R})}=\left\|\left(1+|k|^{2}\right)^{\frac{s}{2}} \hat{u}\right\|_{L^{2}(\mathbb{R})} .
$$

If we denote

$$
\hat{F}\left(k+k_{2}\right)=\int_{\mathbb{R}}\left|\hat{v}\left(k_{1}\right)\right|\left|\hat{v}\left(k-k_{1}+k_{2}\right)\right| d k_{1}=|\hat{v}| *|\hat{v}|
$$


then (14) and (15) applied to $\hat{u}=\hat{w}=|\hat{v}| \in L_{w}^{2}$ yields

$$
\left\|\left.(|\hat{v}| *|\hat{v}|)\right|_{H^{s}(\mathbb{R})} \leq\right\|(|\hat{v}|)\left\|_{H^{s}(\mathbb{R})}^{2}=\right\| v \|_{H^{s}(\mathbb{R})}^{2}
$$

where ` denotes the inverse Fourier transform. Now

$$
\hat{\mathcal{I}}\{v\}=\int_{\mathbb{R}}\left|\hat{v}\left(k_{2}\right)\right| \hat{F}\left(k+k_{2}\right) d k_{2}=|\hat{v}| * \hat{F},
$$

so we apply the above argument to $\hat{u}=|\hat{v}|, \hat{w}=\hat{F}$ to conclude that

$$
\|\mathcal{I}\{v\}\|_{H^{s}(\mathbb{R})} \leq\|v\|_{H^{s}(\mathbb{R})}^{3} .
$$

A standard extension of this argument shows that $\langle C\rangle\{\cdot\}$ is locally Lipschitz on $H^{s}(\mathbb{R})$ for $s>\frac{1}{2}$.

$$
\|\langle C\rangle\{u-v\}\|_{H^{s}(\mathbb{R})} \leq M\|u-v\|_{H^{s}(\mathbb{R})}
$$

where $M$ depends on $\|u\|_{H^{s}(\mathbb{R})}$ and $\|v\|_{H^{s}(\mathbb{R})}$.

\subsection{Well posedness}

The averaged equation (7) is similar in form to the focusing NLS equation, and local well posedness is a straightforward application of semigroup theory.

Theorem 2.2.1 If $v_{0} \in H^{s}(\mathbb{R}), s>\frac{1}{2}$, then there exists $z_{\max }>0$ and $a$ unique solution $v(z, t) \in C\left(\left[0, z_{\max }\right), H^{s}(\mathbb{R})\right)$ for (7) with initial data $v_{0}$, with the property that either $z_{\max }=\infty$ or $z_{\max }<\infty$ and $\lim _{z \rightarrow z_{\max }}\|v(z)\|_{H^{s}}=\infty$.

Proof :

The linear part can be solved via Fourier transform, generating a $C_{0}$ group of unitary operators $\mathcal{S}(z)$ on $H^{s}(\mathbb{R})$ for $z \in \mathbb{R}$. Since $\langle C\rangle\{\cdot\}$ is locally Lipschitz from $H^{s}(\mathbb{R}) \rightarrow H^{s}(\mathbb{R})$, local existence follows [33].

To prove a global existence theorem, a priori estimates on solutions of (7) of the form $\|v(z)\|_{H^{s}(\mathbb{R})}<C(z)$ for any $z \in \mathbb{R}^{+}$are needed. This is possible for initial data in $H^{s}(\mathbb{R}), s \geq 1$, using conservation of the $L^{2}$ norm, conservation of the Hamiltonian, and regularity of the operator $\langle C\rangle$.

Theorem 2.2.2 If $v_{0} \in H^{s}(\mathbb{R}), s \geq 1$, then there exists a unique solution $v(z, t) \in C\left([0, \infty), H^{s}(\mathbb{R})\right)$ for (7) with initial data $v_{0}$.

Proof : 
Multiplying (9) by $\overline{\hat{v}}$, its conjugate by $\hat{v}$, subtracting, and integrating over $\mathbb{R}$ yields

$$
\partial_{z} \int_{\mathbb{R}}|\hat{v}|^{2} d k=-2 \operatorname{Im} \int_{\mathbb{R}} \overline{\hat{v}}\langle\hat{C}\rangle\{v\} d k
$$

where

$$
\begin{gathered}
\int_{\mathbb{R}} \overline{\hat{v}}\langle\hat{C}\rangle\{v\} d k=\int_{\mathbb{R}} \hat{\bar{v}} \int_{\mathbb{R}^{3}} \delta\left(k-k_{1}+k_{2}-k_{3}\right) \Theta_{l}\left(\Delta_{2}, \Delta_{3}\right) \hat{v}_{1} \overline{\hat{v}}_{2} \hat{v}_{3} d k_{1} d k_{2} d k_{3} d k= \\
\int_{\mathbb{R}} \int_{\mathbb{R}^{3}} \delta\left(k-k_{1}+k_{2}-k_{3}\right) \Theta_{l}\left(\Delta_{2}, \Delta_{3}\right) \overline{\hat{v}} \hat{v}_{1} \overline{\hat{v}}_{2} \hat{v}_{3} d k_{1} d k_{2} d k_{3} d k
\end{gathered}
$$

with

$$
\begin{gathered}
\Delta_{2}=k_{1}^{2}-k_{2}^{2}+k_{3}^{2}-k^{2} \\
\Delta_{3}=k_{1}^{3}-k_{2}^{3}+k_{3}^{3}-k^{3} .
\end{gathered}
$$

Since $\overline{\Theta_{l}\left(\Delta_{2}, \Delta_{3}\right)}=\Theta_{l}\left(-\Delta_{2},-\Delta_{3}\right)$, making the change of variables $k \rightarrow k_{3}$, $k_{1} \rightarrow k_{2}$ we see that

$$
\begin{gathered}
\int_{\mathbb{R}} \int_{\mathbb{R}^{3}} \delta\left(k-k_{1}+k_{2}-k_{3}\right) \Theta_{l}\left(\Delta_{2}, \Delta_{3}\right) \overline{\hat{v}} \hat{v}_{1}(z) \overline{\hat{v}}_{2}(z) \hat{v}_{3}(z) d k_{1} d k_{2} d k_{3} d k= \\
\int_{\mathbb{R}} \int_{\mathbb{R}^{3}} \delta\left(k-k_{1}+k_{2}-k_{3}\right) \Theta_{l}\left(\Delta_{2}, \Delta_{3}\right) \overline{\hat{v}} \hat{v}_{1}(z) \overline{\hat{v}}_{2}(z) \hat{v}_{3}(z) d k_{1} d k_{2} d k_{3} d k,
\end{gathered}
$$

so that

$$
\partial_{z} \int_{\mathbb{R}}|v|^{2}=\partial_{z} \int_{\mathbb{R}}|\hat{v}|^{2} d k=0
$$

and the $L^{2}$ norm is conserved.

By conservation of the Hamiltonian,

$$
\begin{gathered}
\langle H\rangle\left(v_{0}\right)=\alpha_{2} \int_{\mathbb{R}}\left|\frac{\partial v_{0}}{\partial t}\right|^{2} d t-\frac{1}{2} \int_{0}^{1} \int_{\mathbb{R}}\left|\mathcal{L}\left(z^{\prime}\right)\left\{v_{0}\right\}\right|^{4} d t d z^{\prime}= \\
\langle H\rangle(v)=\alpha_{2} \int_{\mathbb{R}}\left|\frac{\partial v}{\partial t}\right|^{2} d t-\frac{1}{2} \int_{0}^{1} \int_{\mathbb{R}}\left|\mathcal{L}\left(z^{\prime}\right)\{v\}\right|^{4} d t d z^{\prime} .
\end{gathered}
$$

Thus

$$
\begin{aligned}
\int_{\mathbb{R}}\left|\frac{\partial v}{\partial t}\right|^{2} d t= & \frac{\langle H\rangle\left(v_{0}\right)}{\alpha_{2}}+\frac{1}{2 \alpha_{2}} \int_{0}^{1} \int_{\mathbb{R}}\left|\mathcal{L}\left(z^{\prime}\right)\{v\}\right|^{4} d t d z^{\prime} \\
& \leq \frac{\langle H\rangle\left(v_{0}\right)}{\alpha_{2}}+\frac{M}{2 \alpha_{2}}\|v\|_{L^{2}(\mathbb{R})}^{3 / 2} \cdot\left\|\frac{\partial v}{\partial t}\right\|_{L^{2}(\mathbb{R})}
\end{aligned}
$$


by the Sobolev inequality. From standard estimates [35],

$$
\int_{\mathbb{R}}\left|\frac{\partial v}{\partial t}\right|^{2} d t \leq M^{\prime}
$$

At this point we note that the boundedness of $\|v(z)\|_{H^{1}}$ implies the boundedness of $\|v(z)\|_{L^{p}}$ for $p \geq 2$.

We complete the proof by demonstrating that $\|v(z)\|_{H^{s}(\mathbb{R})}<M(z)$. We start with the integral formulation of (7)

$$
v(z, t)=\mathcal{S}(z)\left\{v_{0}\right\}-\int_{0}^{z} \mathcal{S}\left(z-z^{\prime}\right)\left\{\langle C\rangle\left\{v\left(z^{\prime}\right)\right\}\right\} d z^{\prime}
$$

so that for $s \geq 1$,

$$
\begin{array}{r}
\|v(z)\|_{H^{s}(\mathbb{R})} \leq\left\|\mathcal{S}(z)\left\{v_{0}\right\}\right\|_{H^{s}(\mathbb{R})}+\int_{0}^{z}\left\|\mathcal{S}\left(z-z^{\prime}\right)\left\{\langle C\rangle\left\{v\left(z^{\prime}\right)\right\}\right\}\right\|_{H^{s}(\mathbb{R})} d z^{\prime} \\
=\left\|v_{0}\right\|_{H^{s}(\mathbb{R})}+\int_{0}^{z}\left\|\langle C\rangle\left\{v\left(z^{\prime}\right)\right\}\right\|_{H^{s}(\mathbb{R})} d z^{\prime} \\
\leq\left\|v_{0}\right\|_{H^{s}(\mathbb{R})}+\int_{0}^{z}\left\|v\left(z^{\prime}\right)\right\|_{H^{s}(\mathbb{R})}^{3} d z^{\prime} \\
\leq\left\|v_{0}\right\|_{H^{s}(\mathbb{R})}+\int_{0}^{z}\left\|v\left(z^{\prime}\right)\right\|_{L^{\infty}(\mathbb{R})}^{2}\left\|v\left(z^{\prime}\right)\right\|_{H^{s}(\mathbb{R})} d z^{\prime} \\
\leq\left\|v_{0}\right\|_{H^{s}(\mathbb{R})}+M^{\prime} \int_{0}^{z}\left\|v\left(z^{\prime}\right)\right\|_{H^{s}(\mathbb{R})} d z^{\prime}
\end{array}
$$

Gronwall's inequality gives that $\|v(z)\|_{H^{s}(\mathbb{R})}$ is bounded on $[0, z)$, and this, in combination with the local well posedness result, gives global existence [30].

\subsection{Averaging theorem}

For this section it is most convenient to rescale $z \rightarrow \frac{z}{\epsilon}$ in the transformed and averaged equations (5) and (7) so that

$$
i \frac{\partial v^{\epsilon}}{\partial z}+\alpha_{2} \frac{\partial^{2} v^{\epsilon}}{\partial t^{2}}+C\left(\frac{z}{\epsilon}\right)\left\{v^{\epsilon}\right\}=0
$$

and

$$
i \frac{\partial v}{\partial z}+\alpha_{2} \frac{\partial^{2} v}{\partial t^{2}}+\langle C\rangle\{v\}=0
$$

The validity of the averaging procedure is addressed in the following theorem. 
Theorem 2.3.1 Let $v(z, t) \in C^{0}\left(\left[0, z^{*}\right], H^{s}(\mathbb{R})\right)$ be a solution of (18) on the time interval $\left[0, z^{*}\right]$ for any $z^{*}>0$ and $s>\frac{7}{2}$. Then for $\epsilon$ sufficiently small, there exists $v^{\epsilon}(z, t)$ a solution of (17) with initial data $v(0, t)$ such that $\left\|v^{\epsilon}-v\right\|_{L^{\infty}\left(\left[0, \frac{z^{*}}{\epsilon}\right], H^{s-3}(\mathbb{R})\right)}<C \epsilon$.

Remark : We note that since $u=\mathcal{L}\left\{v^{\epsilon}\right\}$, the standard averaging result

$$
\|u-\mathcal{L}\{v\}\|_{L^{\infty}\left(\left[0, \frac{z^{*}}{\epsilon}\right], H^{s-3}(\mathbb{R})\right)}<\epsilon
$$

follows immediately by isometry.

Proof :

The proof is similar in spirit to classical averaging results in finite dimensions [32], and follows closely the method of [38]. We first split $C\left(\frac{z}{\epsilon}\right)\{v\}$ into its average and varying components

$$
C\left(\frac{z}{\epsilon}\right)\{v\}=\langle C\rangle\{v\}+\mathcal{R}\left(\frac{z}{\epsilon}\right)\{v\}
$$

where

$\hat{\mathcal{R}}\left(\frac{z}{\epsilon}\right)\{v\}=\int_{\mathbb{R}^{3}} \delta\left(k-k_{1}+k_{2}-k_{3}\right) \mathcal{A}\left(\frac{z}{\epsilon}, k, k_{1}, k_{2}, k_{3}\right) \hat{v}\left(z, k_{1}\right) \overline{\hat{v}}\left(z, k_{2}\right) \hat{v}\left(z, k_{3}\right) d k_{1} d k_{2} d k_{3}$

and

$$
\mathcal{A}\left(\frac{z}{\epsilon}, k, k_{1}, k_{2}, k_{3}\right)=\Theta\left(\frac{z}{\epsilon}, k, k_{1}, k_{2}, k_{3}\right)-\Theta_{l}\left(k, k_{1}, k_{2}, k_{3}\right) .
$$

The function $\mathcal{A}$ is bounded in its spatial variables, uniformly in $z$ and $\epsilon$. Now consider

$\mathcal{B}_{\epsilon}\left(z, k, k_{1}, k_{2}, k_{3}\right)=\int_{0}^{z} \mathcal{A}\left(\frac{\tau}{\epsilon}, k, k_{1}, k_{2}, k_{3}\right) d \tau=\epsilon \int_{0}^{\frac{z}{\epsilon}} \mathcal{A}\left(\tau^{\prime}, k, k_{1}, k_{2}, k_{3}\right) d \tau^{\prime}$

where $\tau^{\prime}=\frac{\tau}{\epsilon}$. Since the integrand is 1 - periodic with zero mean, we may write

$$
\epsilon \int_{0}^{\frac{z}{\epsilon}} \mathcal{A}\left(\tau^{\prime}, k, k_{1}, k_{2}, k_{3}\right) d \tau^{\prime}=\epsilon \int_{0}^{z^{\prime \prime}} \mathcal{A}\left(\tau^{\prime}, k, k_{1}, k_{2}, k_{3}\right) d \tau^{\prime}
$$

where $z^{\prime \prime}=\frac{z}{\epsilon}-\left[\frac{z}{\epsilon}\right] \in[0,1)$, with [.] denoting the greatest integer function. Thus

$$
\left\|\mathcal{B}_{\epsilon}\right\|_{L^{\infty}\left(\mathbb{R}^{5}\right)} \leq \epsilon \int_{0}^{z^{\prime \prime}}\|\mathcal{A}\|_{L^{\infty}\left(\mathbb{R}^{5}\right)} \leq \epsilon z^{\prime \prime}\|\mathcal{A}\|_{L^{\infty}\left(\mathbb{R}^{5}\right)} \leq M \epsilon
$$

where $M$ is independent of $z$.

We define the local average $\tilde{v}=v+v_{1}$, where $\hat{v}_{1}(z, k)=i \int_{\mathbb{R}^{3}} \delta\left(k-k_{1}+k_{2}-k_{3}\right) \mathcal{B}_{\epsilon}\left(z, k, k_{1}, k_{2}, k_{3}\right) \hat{v}\left(z, k_{1}\right) \overline{\hat{v}}\left(z, k_{2}\right) \hat{v}\left(z, k_{3}\right) d k_{1} d k_{2} d k_{3}$. 
By direct estimation

$$
\begin{aligned}
\left|\hat{v}_{1}(z, k)\right|= & \left|i \int_{\mathbb{R}^{3}} \delta\left(k-k_{1}+k_{2}-k_{3}\right) \mathcal{B}_{\epsilon}\left(z, k, k_{1}, k_{2}, k_{3}\right) \hat{v}\left(z, k_{1}\right) \overline{\hat{v}}\left(z, k_{2}\right) \hat{v}\left(z, k_{3}\right) d k_{1} d k_{2} d k_{3}\right| \\
& \leq\left.|| \mathcal{B}_{\epsilon}\right|_{L^{\infty}\left(\mathbb{R}^{5}\right)}\left|\int_{\mathbb{R}^{3}} \delta\left(k-k_{1}+k_{2}-k_{3}\right)\right| \hat{v}\left(z, k_{1}\right)|| \overline{\hat{v}}\left(z, k_{2}\right)|| \hat{v}\left(z, k_{3}\right)\left|d k_{1} d k_{2} d k_{3}\right| .
\end{aligned}
$$

Repeating the arguments for regularity of $\langle C\rangle$ in Section 2.1, we have that for every $\sigma>\frac{1}{2}$,

$$
\left\|v_{1}(z)\right\|_{H^{\sigma}} \leq M \epsilon\|v(z)\|_{H^{\sigma}}^{3}
$$

Moreover, by the energy estimate in the well posedness Theorem 2.2.2, the bound is uniform in $z$ for $s \geq 1$,

$$
\sup _{0 \leq z \leq z^{*}}\left\|v_{1}(z)\right\|_{H^{\sigma}} \leq M \epsilon \sup _{0 \leq z \leq z^{*}}\|v(z)\|_{H^{\sigma}}^{3} \leq M^{\prime} \epsilon .
$$

We directly compute

$$
i \frac{\partial \hat{v}_{1}}{\partial z}=\hat{\mathcal{R}}^{\prime}-\hat{\mathcal{R}}
$$

where

$\hat{\mathcal{R}}^{\prime}=i \int_{\mathbb{R}^{3}} \delta\left(k-k_{1}+k_{2}-k_{3}\right) \mathcal{B}_{\epsilon}\left(z, k, k_{1}, k_{2}, k_{3}\right) \partial_{z}\left\{\hat{v}\left(z, k_{1}\right) \overline{\hat{v}}\left(z, k_{2}\right) \hat{v}\left(z, k_{3}\right)\right\} d k_{1} d k_{2} d k_{3}$.

Using equation (7), we estimate

$$
\left\|\mathcal{R}^{\prime}\right\|_{H^{s-3}} \leq M \epsilon
$$

The local average $\tilde{v}$ satisfies

$$
i \frac{\partial \tilde{v}}{\partial z}+\alpha_{2} \frac{\partial^{2} \tilde{v}}{\partial t^{2}}+C\left(\frac{z}{\epsilon}\right)\{\tilde{v}\}=\mathcal{R}^{\prime \prime},
$$

where

$$
\mathcal{R}^{\prime \prime}=C\left(\frac{z}{\epsilon}\right)\{\tilde{v}\}-C\left(\frac{z}{\epsilon}\right)\{v\}+\mathcal{R}^{\prime}+\alpha_{2} \frac{\partial^{2} v_{1}}{\partial t^{2}} .
$$

By continuity of $C\left(\frac{z}{\epsilon}\right)\{\cdot\}$

$$
\left\|C\left(\frac{z}{\epsilon}\right)\{\tilde{v}\}-C\left(\frac{z}{\epsilon}\right)\{v\}\right\|_{H^{s}} \leq M \epsilon
$$

for all $s>\frac{1}{2}$, and so

$$
\left\|\mathcal{R}^{\prime \prime}\right\|_{H^{s-3}} \leq M \epsilon .
$$

Finally we consider

$$
f_{\epsilon}=v^{\epsilon}-\tilde{v}
$$


which satisfies

$$
i \frac{\partial f_{\epsilon}}{\partial z}+\alpha_{2} \frac{\partial^{2} f_{\epsilon}}{\partial t^{2}}+C\left(\frac{z}{\epsilon}\right)\left\{\tilde{v}+f_{\epsilon}\right\}-C\left(\frac{z}{\epsilon}\right)\{\tilde{v}\}=-\mathcal{R}^{\prime \prime} .
$$

Again by continuity of $C\left(\frac{z}{\epsilon}\right)\{\cdot\}$

$$
\left\|C\left(\frac{z}{\epsilon}\right)\left\{\tilde{v}+f_{\epsilon}\right\}-C\left(\frac{z}{\epsilon}\right)\{\tilde{v}\}\right\|_{H^{s-3}} \leq M\left\|f_{\epsilon}\right\|_{H^{s-3}} .
$$

Writing (19) in Fourier space, multiplying the equation by $\left(1+|k|^{2}\right)^{s-3} \hat{f}_{\epsilon}$, its conjugate by $\left(1+|k|^{2}\right)^{s-3} f_{\epsilon}$, subtracting and integrating over $k$, one obtains

$$
\frac{\partial}{\partial z}\left\|f_{\epsilon}\right\|_{H^{s-3}}^{2} \leq M \epsilon\left\|f_{\epsilon}\right\|_{H^{s-3}}^{2}+M\left\|f_{\epsilon}\right\|_{H^{s-3}}^{4} .
$$

Since $\left\|f_{\epsilon}\right\|_{H^{s-3}}^{2}<M$, we can write the estimate

$$
\frac{\partial}{\partial z}\left\|f_{\epsilon}\right\|_{H^{s-3}}^{2} \leq M^{2} \epsilon+M^{2}\left\|f_{\epsilon}\right\|_{H^{s-3}}^{2} .
$$

Now using the fact that $\left\|f_{\epsilon}(0)\right\|_{H^{s-3}}^{2}=0$ and applying Gronwall's inequality we have

$$
\left\|f_{\epsilon}(z)\right\|_{H^{s-3}} \leq e^{M^{2} \epsilon z} M^{2} \epsilon \leq e^{K} M^{2} \epsilon
$$

where $K$ is a time-independent constant for $z \sim \mathcal{O}\left(\frac{1}{\epsilon}\right)$, so

$$
\sup _{0 \leq z \leq \frac{z^{*}}{\epsilon}}\left\|f_{\epsilon}\right\|_{H^{s-3}} \leq M^{\prime} \epsilon
$$

Overall, we have

$$
\begin{aligned}
\sup _{0 \leq z \leq \frac{z^{*}}{\epsilon}}\left\|v^{\epsilon}-v\right\|_{H^{s-3}} & \leq \sup _{0 \leq z \leq \frac{z^{*}}{\epsilon}}\left\|v^{\epsilon}-\tilde{v}\right\|_{H^{s-3}}+\sup _{0 \leq z \leq \frac{z^{*}}{\epsilon}}\|\tilde{v}-v\|_{H^{s-3}} \\
& =\sup _{0 \leq z \leq \frac{z^{*}}{\epsilon}}\left\|f_{\epsilon}\right\|_{H^{s-3}}+\sup _{0 \leq z \leq \frac{z^{*}}{\epsilon}}\left\|v_{1}(z)\right\|_{H^{s-3}} \leq M \epsilon
\end{aligned}
$$

\section{Existence of a ground state}

Here we show that for the case $\alpha_{2}>0$ and $\alpha_{3}=0$, the averaged Hamiltonian possesses a minimizer in the class of admissible functions $\mathcal{A}_{\lambda}=\left\{v: \int_{\mathbb{R}}|v|^{2}=\right.$ $\left.\lambda, \int_{\mathbb{R}}\left|v_{t}\right|^{2}<\infty\right\}$. We adapt an argument first established for the NLS equation [6] and later adapted for the case of second order dispersion management [38]. We first present properties of the Hamiltonian that are essential to the minimization argument. 


\subsection{Properties of $\langle H\rangle$}

3.1.1 $\inf _{\mathcal{A}_{\lambda}}\langle H\rangle\{v\}<0$

Proof :

We first assume that the 1 - periodic dispersion maps $d_{i}(z), i=2,3$, are piecewise constant and of the following form, which is standard in optical communications

$$
\tilde{d}_{i}(z)= \begin{cases}\tilde{D}_{i} & \text { if } z \in[0, \theta) \text { or } z \in[1-\theta, 1) \\ -\tilde{D}_{i} & \text { if } z \in[\theta, 1-\theta) .\end{cases}
$$

For these dispersion profiles we define the map strength parameters $s_{i}$ by $s_{i}=$ $\theta \tilde{D}_{i}$. The Hamiltonian can be written

$$
\begin{gathered}
\langle H\rangle\{v\}=\alpha_{2} \int_{\mathbb{R}}\left|v_{t}\right|^{2} d t- \\
\frac{1}{2} \int_{0}^{1} \int_{\mathbb{R}} \int_{\mathbb{R}^{4}} e^{i\left(k_{1}-k_{2}+k_{3}-k_{4}\right) t} e^{-i \Delta_{2} \int_{0}^{z} d_{2}\left(z^{\prime}\right)+i \Delta_{3} \int_{0}^{z} d_{3}\left(z^{\prime}\right) d z^{\prime}} \\
\times \hat{v}\left(k_{1}\right) \overline{\hat{v}}\left(k_{2}\right) \hat{v}\left(k_{3}\right) \overline{\hat{v}}\left(k_{4}\right) d k_{1} d k_{2} d k_{3} d k_{4} d t d z
\end{gathered}
$$

where $\Delta_{2}=k_{1}^{2}-k_{2}^{2}+k_{3}^{2}-k_{4}^{2}$ and $\Delta_{3}=k_{1}^{3}-k_{2}^{3}+k_{3}^{3}-k_{4}^{3}$. Performing the integration in $z$ yields

$$
\begin{gathered}
\langle H\rangle\{v\}=\alpha_{2} \int_{\mathbb{R}}\left|v_{t}\right|^{2} d t \\
-\frac{1}{2} \int_{\mathbb{R}} \int_{\mathbb{R}^{4}} e^{i\left(k_{1}-k_{2}+k_{3}-k_{4}\right) t}\left(\frac{\theta \sin \left(s_{2} \Delta_{2}+s_{3} \Delta_{3}\right)}{s_{2} \Delta_{2}+s_{3} \Delta_{3}}\right) \\
\times \hat{v}\left(k_{1}\right) \overline{\hat{v}}\left(k_{2}\right) \hat{v}\left(k_{3}\right) \overline{\hat{v}}\left(k_{4}\right) d k_{1} d k_{2} d k_{3} d k_{4} d t
\end{gathered}
$$

Let $v$ be an arbitrary element of $\mathcal{A}_{\lambda}$ and consider the rescaled function

$$
v_{\gamma}(t)=\gamma^{\frac{1}{2}} v(\gamma t),
$$

which is also an element of $\mathcal{A}_{\lambda}$. A scaling property of the Fourier transform gives that

$$
\hat{v}_{\gamma}(k)=\gamma^{\frac{-1}{2}} \hat{v}\left(\frac{k}{\gamma}\right)
$$

and the chain rule yields

$$
\frac{\partial v_{\gamma}(t)}{\partial t}=\gamma^{\frac{3}{2}} \frac{\partial v\left(t^{\prime}\right)}{\partial t^{\prime}}
$$


where $t^{\prime}=\gamma t$. Substituting $v_{\gamma}$ into the Hamiltonian, we have

$$
\begin{gathered}
F(\gamma)=\langle H\rangle\left\{v_{\gamma}\right\}=\alpha_{2} \int_{\mathbb{R}}\left|\gamma^{\frac{3}{2}} \frac{\partial v\left(t^{\prime}\right)}{\partial t^{\prime}}\right|^{2} d t- \\
\frac{1}{2 \gamma^{2}} \int_{\mathbb{R}} \int_{\mathbb{R}^{4}} e^{i\left(k_{1}-k_{2}+k_{3}-k_{4}\right) t}\left(\frac{\theta \sin \left(s_{2} \Delta_{2}+s_{3} \Delta_{3}\right)}{s_{2} \Delta_{2}+s_{3} \Delta_{3}}\right) \\
\times \hat{v}\left(\frac{k_{1}}{\gamma}\right) \overline{\hat{v}}\left(\frac{k_{2}}{\gamma}\right) \hat{v}\left(\frac{k_{3}}{\gamma}\right) \overline{\hat{v}}\left(\frac{k_{4}}{\gamma}\right) d k_{1} d k_{2} d k_{3} d k_{4} d t \\
=\gamma^{2} \alpha_{2} \int_{\mathbb{R}}\left|\frac{\partial v\left(t^{\prime}\right)}{\partial t^{\prime}}\right|^{2} d t^{\prime}- \\
\frac{1}{2 \gamma^{3}} \int_{\mathbb{R}} \int_{\mathbb{R}^{4}} e^{i\left(\frac{k_{1}}{\gamma}-\frac{k_{2}}{\gamma}+\frac{k_{3}}{\gamma}-\frac{k_{4}}{\gamma}\right) t^{\prime}}\left(\frac{\theta \sin \left(s_{2} \gamma^{2} \tilde{\Delta}_{2}+s_{3} \gamma^{3} \tilde{\Delta}_{3}\right)}{s_{2} \gamma^{2} \tilde{\Delta}_{2}+s_{3} \gamma^{3} \tilde{\Delta}_{3}}\right) \\
\times \hat{v}\left(\frac{k_{1}}{\gamma}\right) \overline{\hat{v}}\left(\frac{k_{2}}{\gamma}\right) \hat{v}\left(\frac{k_{3}}{\gamma}\right) \overline{\hat{v}}\left(\frac{k_{4}}{\gamma}\right) d k_{1} d k_{2} d k_{3} d k_{4} d t^{\prime},
\end{gathered}
$$

where

$$
\tilde{\Delta}_{2}=\left(\frac{k_{1}}{\gamma}\right)^{2}-\left(\frac{k_{2}}{\gamma}\right)^{2}+\left(\frac{k_{3}}{\gamma}\right)^{2}-\left(\frac{k_{4}}{\gamma}\right)^{2}
$$

and

$$
\tilde{\Delta}_{3}=\left(\frac{k_{1}}{\gamma}\right)^{3}-\left(\frac{k_{2}}{\gamma}\right)^{3}+\left(\frac{k_{3}}{\gamma}\right)^{3}-\left(\frac{k_{4}}{\gamma}\right)^{3} .
$$

Making the change of variable $k_{j}^{\prime}=\frac{k_{j}}{\gamma}$, we have

$$
\begin{gathered}
F(\gamma)=C \gamma^{2}- \\
\frac{\gamma}{2} \int_{\mathbb{R}} \int_{\mathbb{R}^{4}} e^{i\left(k_{1}^{\prime}-k_{2}^{\prime}+k_{3}^{\prime}-k_{4}^{\prime}\right) t^{\prime}}\left(\frac{\theta \sin \left(s_{2} \gamma^{2} \Delta_{2}^{\prime}+s_{3} \gamma^{3} \Delta_{3}^{\prime}\right)}{s_{2} \gamma^{2} \Delta_{2}^{\prime}+s_{3} \gamma^{3} \Delta_{3}^{\prime}}\right) \\
\times \hat{v}\left(k_{1}^{\prime}\right) \overline{\hat{v}}\left(k_{2}^{\prime}\right) \hat{v}\left(k_{3}^{\prime}\right) \overline{\hat{v}}\left(k_{4}^{\prime}\right) d k_{1}^{\prime} d k_{2}^{\prime} d k_{3}^{\prime} d k_{4}^{\prime} d t^{\prime} \\
=C \gamma^{2}-\gamma G\left(v ; \gamma, s_{2}, s_{3}, \theta\right)
\end{gathered}
$$


where $\Delta_{2}^{\prime}=\left(k_{1}^{\prime}\right)^{2}-\left(k_{2}^{\prime}\right)^{2}+\left(k_{3}^{\prime}\right)^{2}-\left(k_{4}^{\prime}\right)^{2}, \Delta_{3}^{\prime}=\left(k_{1}^{\prime}\right)^{3}-\left(k_{2}^{\prime}\right)^{3}+\left(k_{3}^{\prime}\right)^{3}-\left(k_{4}^{\prime}\right)^{3}$, and $G$ is a functional of $v$ also depending on $\gamma, s_{2}, s_{3}$ and $\theta$. At this stage, we can see that as $s_{j} \rightarrow 0$ and $\theta \rightarrow 1$, we recover the exact scaling of the integrable NLS Hamiltonian evaluated at $v_{\gamma}$. To show that this Hamiltonian can be made negative, we first note that by continuity of the kernel

$$
K\left(\gamma, \theta, s_{j}, \Delta_{j}^{\prime}\right)=\left(\frac{\theta \sin \left(s_{2} \gamma^{2} \Delta_{2}^{\prime}+s_{3} \gamma^{3} \Delta_{3}^{\prime}\right)}{s_{2} \gamma^{2} \Delta_{2}^{\prime}+s_{3} \gamma^{3} \Delta_{3}^{\prime}}\right)
$$

in $\gamma, F(0)=0$. Moreover, differentiating the functional $F$ in $\gamma$ yields

$$
F^{\prime}(\gamma)=2 C \gamma-\gamma G^{\prime}\left(v ; \gamma, s_{2}, s_{3}, \theta\right)-G\left(v ; \gamma, s_{2}, s_{3}, \theta\right)
$$

To compute $G^{\prime}$, we differentiate under the integral sign and apply the chain rule. This gives $G^{\prime}\left(v ; 0, s_{2}, s_{3}, \theta\right)=0$, so that

$$
\begin{aligned}
F^{\prime}(0)= & -G\left(v ; 0, s_{2}, s_{3}, \theta\right)=-\frac{1}{2} \int_{\mathbb{R}} \int_{\mathbb{R}^{4}} e^{i\left(k_{1}^{\prime}-k_{2}^{\prime}+k_{3}^{\prime}-k_{4}^{\prime}\right) t^{\prime}} \\
& \times \hat{v}\left(k_{1}^{\prime}\right) \overline{\hat{v}}\left(k_{2}^{\prime}\right) \hat{v}\left(k_{3}^{\prime}\right) \overline{\hat{v}}\left(k_{4}^{\prime}\right) d k_{1}^{\prime} d k_{2}^{\prime} d k_{3}^{\prime} d k_{4}^{\prime} d t^{\prime} \\
& =-\frac{1}{2} \int_{\mathbb{R}}\left|v\left(t^{\prime}\right)\right|^{4} d t^{\prime}<0 .
\end{aligned}
$$

Thus for $\gamma$ small enough, the Hamiltonian is negative.

\subsection{2 $\langle H\rangle\{v\}$ is subadditive :}

If $I_{\lambda}=\inf _{v \in \mathcal{A}_{\lambda}}\langle H\rangle\{v\}$, then $I_{\lambda_{1}+\lambda_{2}}<I_{\lambda_{1}}+I_{\lambda_{2}}$ Claim : For $\theta>1, I_{\theta \lambda}<\theta I_{\lambda}$

Proof of claim :

$$
\begin{aligned}
& I_{\theta \lambda}=\inf _{v \in \mathcal{A}_{\theta \lambda}}\langle H\rangle\{v\} \\
& =\inf _{w \in \mathcal{A}_{\lambda}}\langle H\rangle\{\sqrt{\theta} w\}
\end{aligned}
$$

since

$$
\|w\|_{L^{2}}^{2}=\lambda \Rightarrow\|\sqrt{\theta} w\|_{L^{2}}^{2}=\theta \lambda .
$$

But

$$
\begin{gathered}
\langle H\rangle(\sqrt{\theta} w)=\alpha_{2} \int_{\mathbb{R}}\left|(\sqrt{\theta} w)_{t}\right|^{2} d t-\frac{1}{2} \int_{0}^{1} \int_{\mathbb{R}}|\mathcal{L}(z)\{\sqrt{\theta} w\}|^{4} d t d z \\
=\theta \alpha_{2} \int_{\mathbb{R}}\left|w_{t}\right|^{2} d t-\frac{\theta^{2}}{2} \int_{0}^{1} \int_{\mathbb{R}}|\mathcal{L}(z)\{w\}|^{4} d t d z
\end{gathered}
$$




$$
<\theta\left(\alpha_{2} \int_{\mathbb{R}}\left|w_{t}\right|^{2} d t-\frac{1}{2} \int_{0}^{1} \int_{\mathbb{R}} \int_{\mathbb{R}}|w|^{4} d t d z\right)
$$

for $\theta>1$. So

$$
I_{\theta \lambda}=\inf _{w \in \mathcal{A}_{\lambda}}\langle H\rangle\{\sqrt{\theta} w\}<\theta \inf _{w \in \mathcal{A}_{\lambda}}\langle H\rangle\{w\}=\theta I_{\lambda} .
$$

Proof of subadditivity:

If we set $\lambda_{1}=\alpha \lambda_{2}$ with $\alpha<1$, we have

$$
I_{\lambda_{1}+\lambda_{2}}=I_{\alpha \lambda_{2}+\lambda_{2}}<(\alpha+1) I_{\lambda_{2}}=\alpha I_{\left(\alpha^{-1} \lambda_{1}\right)}+I_{\lambda_{2}}<I_{\lambda_{1}}+I_{\lambda_{2}} .
$$

\subsubsection{Localization of minimizing sequences}

In the minimization proof, we used the fact that for a minimizing sequence $v_{k}(t) \in H^{1}(\mathbb{R})$, there exists a subsequence $v_{k_{m}}(t)$ which remains localized. That is, for any $\epsilon>0$ there exists an $R>0$ such that

$$
\int_{-R}^{+R}\left|w_{m}(t)\right|^{2} d t>\lambda-\epsilon
$$

where $w_{m}(t)=v_{k_{m}}\left(t-t_{m}\right)$ and $\lambda=\int_{\mathbb{R}}\left|w_{m}(t)\right|^{2} d t$. To prove this result, we apply a version of Lions' concentration-compactness lemma [17, 38].

Lemma 3.1.1 If $u_{m} \in H^{1}(\mathbb{R})$ is a bounded sequence with $\left\|u_{m}\right\|_{L^{2}}=\lambda$, then there exists a subsequence $u_{m_{k}}$ for which one of the following properties hold

1. (localization) There exists a sequence $t_{k}$ such that for any $\epsilon>0$ there exists $R>0$ and

$$
\int_{t_{k}-R}^{t_{k}+R}\left|u_{m_{k}}\right|^{2} d x \geq \lambda-\epsilon
$$

2. (vanishing) For any $R>0$

$$
\lim _{k \rightarrow \infty} \sup _{y \in \mathbb{R}} \int_{y-R}^{y+R}\left|u_{m_{k}}\right|^{2} d x \rightarrow 0 .
$$

3. (splitting) There exists $0<\gamma<\lambda$ such that for any $\epsilon>0$, there exist $k_{0}$ and two sequences $v_{k}, w_{k}$ with compact support so that for $k \geq k_{0}$

$$
\begin{aligned}
& \left\|v_{k}\right\|_{H^{1}}+\left\|w_{k}\right\|_{H^{1}} \leq 4 \sup _{k \in \mathbf{N}}\left\|u_{m_{k}}\right\|_{H^{1}} \\
& \left\|u_{m_{k}}-\left(v_{k}+w_{k}\right)\right\|_{L^{2}} \leq 2 \epsilon \\
& \left\|\left|v _ { k } \left\|_{L^{2}}-\gamma\left|\leq \epsilon\left\|v_{k}\right\|_{L^{2}}-(\lambda-\gamma)\right| \leq \epsilon\right.\right.\right. \\
& \left\|\frac{\partial v_{k}}{\partial x}\right\|_{L^{2}}+\left\|\frac{\partial w_{k}}{\partial x}\right\|_{L^{2}} \leq\left\|\frac{\partial u_{m_{k}}}{\partial x}\right\|_{L^{2}}+\epsilon
\end{aligned}
$$

and $\operatorname{dist}\left(\operatorname{supp}\left(v_{k}\right), \operatorname{supp}\left(w_{k}\right)\right)>2 \epsilon^{-1}$. 
Thus, for the minimization problem there exists a localized subsequence of the minimizing sequence if vanishing and splitting can be ruled out.

We first rule out vanishing. Let $v_{k}$ be a minimizing sequence for $\langle H\rangle\{v\}$ and assume that a subsequence $v_{m_{k}}$ vanishes. Since $v_{k}$ is a minimizing sequence, for some $k$ we have

$$
\alpha_{2} \int_{\mathbb{R}}\left|\frac{\partial v_{k}}{\partial t}\right|^{2} d t-\frac{1}{2} \int_{0}^{1} \int_{\mathbb{R}}\left|\mathcal{L}\left(z^{\prime}\right)\left\{v_{k}\right\}\right|^{4} d t d z^{\prime}<0
$$

so that

$$
\int_{0}^{1} \int_{\mathbb{R}}\left|\mathcal{L}\left(z^{\prime}\right)\left\{v_{k}\right\}\right|^{4} d t d z^{\prime}>0
$$

Thus for some $z^{*}$, we have

$$
\int_{\mathbb{R}}\left|\mathcal{L}\left(z^{*}\right)\left\{v_{k}\right\}\right|^{4} d t>0
$$

Applying a lemma of Cazenave [6] for arbitrary $H^{1}(\mathbb{R})$ functions

$$
\int_{\mathbb{R}}|u|^{4} d t \leq C\|u\|_{H^{1}}^{2} \sup _{y \in \mathbb{R}} \int_{y-1}^{y+1}|u|^{2} d t
$$

gives that

$$
\sup _{y \in \mathbb{R}} \int_{y-1}^{y+1}\left|\mathcal{L}\left(z^{*}\right)\left\{v_{k}\right\}\right|^{2} d t>0
$$

Now we relate $\sup _{y \in \mathbb{R}} \int_{y-1}^{y+1}\left|\mathcal{L}\left(z^{*}\right)\left\{v_{k}\right\}\right|^{2} d t$ to $\sup _{y \in \mathbb{R}} \int_{y-1}^{y+1}\left|v_{k}\right|^{2} d t$ with the following localization lemma, which is similar to the lemma of [38].

Lemma 3.1.2 Consider the following linear dispersive equation

$$
i u_{z}+\tilde{d}_{2}(z) u_{t t}+i \tilde{d}_{3}(z) u_{t t t}=0
$$

with $u \in H^{1}(\mathbb{R}),\|u\|_{L^{2}(\mathbb{R})}=1$, and $\tilde{d}_{i}(z)$ piecewise constant. Let $u_{n}(t, z)$ be a sequence of solutions of (24) and define

$$
\epsilon_{n}(z)=\sup _{y \in \mathbb{R}} \int_{y-1}^{y+1}\left|u_{n}(t, z)\right|^{2}
$$

If $u_{n}(t, 0)$ is vanishing initial data $\left(\lim _{n \rightarrow \infty} \epsilon_{n}(0)=0\right)$ with the constraint $\left\|u_{n}\right\|_{L^{2}(\mathbb{R})}=1$, then the sequence of the solutions $u_{n}(t, z)$ is also vanishing $\left(\lim _{n \rightarrow \infty} \epsilon_{n}(z)=0\right)$. 
Proof:

Let $\chi_{m}(t)$ be a smooth approximation to the characteristic function on the interval $[-m, m]$, with the property that $\left|\partial_{t} \chi_{m}\right|<\frac{C}{m}, \chi_{m}(t)=1$ if $|t| \leq 1$, and $\chi(t)=0$ if $|t| \geq m$. Multiplying (24) by $\bar{u} \chi_{m}(t)$, its conjugate by $u \chi_{m}(t)$, subtracting and integrating over $t$ yields

$$
\frac{d}{d z} \int_{\mathbb{R}} \chi_{m}|u|^{2} d t=-2 d_{2}(z) \operatorname{Im} \int_{\mathbb{R}} \chi_{m} \bar{u} u_{t t} d t-2 d_{3}(z) \operatorname{Re} \int_{\mathbb{R}} \chi_{m} \bar{u} u_{t t t} d t
$$

Now

$$
\operatorname{Im} \int_{\mathbb{R}} \chi_{m} \bar{u} u_{t t} d t=-\operatorname{Im} \int_{\mathbb{R}}\left(\chi_{m} \bar{u}_{t}+\chi_{m}^{\prime} \bar{u}\right) u_{t} d t=-\operatorname{Im} \int_{\mathbb{R}} \chi_{m}^{\prime} \bar{u} u_{t} d t
$$

and

$\operatorname{Re} \int_{\mathbb{R}} \chi_{m} \bar{u} u_{t t t} d t=-\operatorname{Re} \int_{\mathbb{R}}\left(\chi_{m} \bar{u}_{t}+\chi_{m}^{\prime} \bar{u}\right) u_{t t} d t=-\operatorname{Re} \int_{\mathbb{R}} \chi_{m} \bar{u}_{t} u_{t t} d t-\operatorname{Re} \int_{\mathbb{R}} \chi_{m}^{\prime} \bar{u} u_{t t} d t$

$=\frac{1}{2} \int_{\mathbb{R}} \frac{d\left|u_{t}\right|^{2}}{d t} \chi_{m}+\operatorname{Re} \int_{\mathbb{R}}\left(\chi_{m}^{\prime \prime} \bar{u}+\chi_{m}^{\prime} \bar{u}_{t}\right) u_{t} d t=\frac{3}{2} \int_{\mathbb{R}}\left|u_{t}\right|^{2} \chi_{m}^{\prime} d t+R e \int_{\mathbb{R}} \chi_{m}^{\prime \prime} \bar{u} u_{t} d t$

Overall,

$\frac{d}{d z} \int_{\mathbb{R}} \chi_{m}|u|^{2} d t=2 d_{2}(z) \operatorname{Im} \int_{\mathbb{R}} \chi_{m}^{\prime} \bar{u} u_{t} d t-2 d_{3}(z)\left(\frac{3}{2} \int_{\mathbb{R}}\left|u_{t}\right|^{2} \chi_{m}^{\prime} d t+\operatorname{Re} \int_{\mathbb{R}} \chi_{m}^{\prime \prime} \bar{u} u_{t} d t\right)$

and integrating from 0 to $z$ gives

$$
\begin{gathered}
\int_{\mathbb{R}} \chi_{m}|u(z)|^{2} d t=\int_{\mathbb{R}} \chi_{m}|u(0)|^{2} d t+\int_{0}^{z}\left(2 d_{2}\left(z^{\prime}\right) \operatorname{Im} \int_{\mathbb{R}} \chi_{m}^{\prime} \bar{u} u_{t} d t+2 d_{3}\left(z^{\prime}\right)\left(\frac{3}{2} \int_{\mathbb{R}}\left|u_{t}\right|^{2} \chi_{m}^{\prime} d t+\operatorname{Re} \int_{\mathbb{R}} \chi_{m}^{\prime \prime} \bar{u} u_{t} d t\right)\right) d z^{\prime} \\
\leq \int_{\mathbb{R}} \chi_{m}|u(0)|^{2} d t+C_{m}\left(\|u\|_{H^{1}},\left\|\chi_{m}^{\prime}\right\|_{L^{\infty}}\left\|\chi_{m}^{\prime \prime}\right\|_{L^{\infty}},\left\|d_{j}\right\|_{L^{\infty}}\right)
\end{gathered}
$$

where $C_{m} \rightarrow 0$ as $m \rightarrow \infty$.

Let $u_{n}(t, z)$ denote a sequence of solutions of (24) with vanishing initial data i.e.

$$
\epsilon_{n}(0)=\sup _{y \in \mathbb{R}} \int_{y-1}^{y+1}\left|u_{n}(t, 0)\right|^{2} \rightarrow 0
$$

If $\epsilon_{n}(z)<\epsilon_{n}(0)$ then we are done, so let $\epsilon_{n}(z)>\epsilon_{n}(0)$. Choosing $\chi_{m_{n}}\left(*-t_{n}\right)$ such that it is centered with respect to $u_{n}(z, t)$, we have

$$
\int_{\mathbb{R}} \chi_{m_{n}}\left|u_{n}(t, z)\right|^{2} d t \geq \epsilon_{n}(z)
$$


and also

$$
\int_{\mathbb{R}} \chi_{m_{n}}\left|u_{n}(t, 0)\right|^{2} d t \leq 2 m_{n} \epsilon_{n}(0) .
$$

and taking the limit $m_{n} \rightarrow \infty$ with $m_{n} \sim \sqrt{\frac{1}{\epsilon_{n}(0)}}$ gives the result.

Returning to (23) and applying the contrapositive of the localization lemma, we have

$$
\sup _{y \in \mathbb{R}} \int_{y-1}^{y+1}\left|v_{k}\right|^{2} d t>0
$$

contradicting the assumption that a subsequence $v_{m_{k}}$ vanishes.

To rule out splitting, it is enough to show that

$$
\langle H\rangle\left\{v_{m_{k}}\right\}>\langle H\rangle\left\{w_{k}\right\}+\langle H\rangle\left\{u_{k}\right\}+\alpha(\epsilon)
$$

where $\alpha(\epsilon)$ is independent of $k$ and goes to 0 as $\epsilon \rightarrow 0$, as this causes $\langle H\rangle\left\{v_{m_{k}}\right\}$ to violate subadditivity. We directly evaluate $\langle H\rangle\left\{v_{m_{k}}\right\}$

$$
\begin{gathered}
\langle H\rangle\left\{v_{m_{k}}\right\}=\alpha_{2} \int_{\mathbb{R}}\left|\frac{\partial\left(u_{k}+w_{k}+h_{k}\right)}{\partial t}\right|^{2} d t \\
-\frac{1}{2} \int_{0}^{1} \int_{\mathbb{R}}\left|\mathcal{L}\left\{u_{k}+w_{k}+h_{k}\right\}\right|^{4} d t d z
\end{gathered}
$$

where

$$
\left\|h_{k}\right\|_{L^{2}}^{2}<\epsilon
$$

and we have suppressed the notation $\mathcal{L}(z)$.

Expanding the terms, this can be rewritten

$$
\begin{array}{r}
\langle H\rangle\left\{v_{m_{k}}\right\}=\langle H\rangle\left\{u_{k}\right\}+\langle H\rangle\left\{w_{k}\right\} \\
+2 \alpha_{2} \operatorname{Re} \int_{\mathbb{R}}\left(\partial_{t} \bar{u}_{k} \partial_{t} w_{k}+\partial_{t} \bar{u}_{k} \partial_{t} h_{k}+\partial_{t} \bar{w}_{k} \partial_{t} h_{k}+\left|\partial_{t} h_{k}\right|^{2}\right) d t \\
-\operatorname{Re} \int_{0}^{1} \int_{\mathbb{R}}\left(\left|\mathcal{L}\left\{u_{k}+w_{k}\right\}\right|^{2}\left|\mathcal{L}\left\{h_{k}\right\}\right|^{2}+\frac{1}{2}\left|\mathcal{L}\left\{h_{k}\right\}\right|^{4}\right. \\
+2\left|\mathcal{L}\left\{u_{k}+w_{k}\right\}\right|^{2}\left(\mathcal{L}\left\{u_{k}+w_{k}\right\}\right)\left(\mathcal{L}\left\{h_{k}\right\}\right)+\left(\mathcal{L}\left\{u_{k}+w_{k}\right\}\right)^{2}\left(\overline{\mathcal{L}\left\{h_{k}\right\}}\right)^{2} \\
\left.+2 \mathcal{L}\left\{u_{k}+w_{k}\right\}\left|\mathcal{L}\left\{h_{k}\right\}\right|^{2} \overline{\mathcal{L} h_{k}}\right) d t d z
\end{array}
$$




$$
\begin{aligned}
& +\frac{1}{2} \int_{0}^{1} \int_{\mathbb{R}}\left(2\left|\mathcal{L}\left\{u_{k}\right\}\right|^{2}\left|\mathcal{L}\left\{w_{k}\right\}\right|^{2}+2\left|\mathcal{L}\left\{u_{k}\right\}\right|^{2} \mathcal{L}\left\{u_{k}\right\} \mathcal{L}\left\{\bar{w}_{k}\right\}\right. \\
& \left.\quad+\left(\mathcal{L}\left\{u_{k}\right\}\right)^{2}\left(\mathcal{L}\left\{\bar{w}_{k}\right\}\right)^{2}+2\left|\mathcal{L}\left\{w_{k}\right\}\right|^{2} \mathcal{L}\left\{u_{k}\right\} \mathcal{L}\left\{\bar{w}_{k}\right\}\right) d t d z .
\end{aligned}
$$

We proceed exactly as in [38]. The terms

$$
2 \alpha_{2} R e \int_{\mathbb{R}}\left(\partial_{t} \bar{u}_{k} \partial_{t} w_{k}+\partial_{t} \bar{u}_{k} \partial_{t} h_{k}+\partial_{t} \bar{w}_{k} \partial_{t} h_{k}+\left|\partial_{t} h_{k}\right|^{2}\right) d t
$$

can be estimated from below by $-C_{1} \epsilon$, with $C_{1}$ depending only on $\lambda$ and $\alpha_{2}$. The terms

$$
\begin{array}{r}
\operatorname{Re} \int_{0}^{1} \int_{\mathbb{R}}\left(2\left|\mathcal{L}\left\{u_{k}+w_{k}\right\}\right|^{2}\left|\mathcal{L}\left\{h_{k}\right\}\right|^{2}+\frac{1}{2}\left|\mathcal{L}\left\{h_{k}\right\}\right|^{4}\right. \\
\left.+2\left|\mathcal{L}\left\{u_{k}+w_{k}\right\}\right|^{2}\left(\mathcal{L}\left\{u_{k}+w_{k}\right\}\right)\left(\mathcal{L}\left\{h_{k}\right\}\right)+\left(\mathcal{L}\left\{u_{k}+w_{k}\right\}\right)^{2} \overline{\left(\mathcal{L}\left\{h_{k}\right\}\right.}\right)^{2} \\
\left.+2 \mathcal{L}\left\{u_{k}+w_{k}\right\}\left|\mathcal{L}\left\{h_{k}\right\}\right|^{2} \overline{\mathcal{L} h h_{k}}\right) d t d z
\end{array}
$$

are all estimated by Holder's inequality and the Sobolev inequality

$$
\int_{0}^{1} \int_{\mathbb{R}}|\mathcal{L}(z)\{v\}|^{4} d t d z \leq M\|v(t)\|_{L^{2}(\mathbb{R})}^{3}\left\|v_{t}(t)\right\|_{L^{2}(\mathbb{R})},
$$

yielding a lower bound of the form $-C_{2}(\epsilon)$. The remaining terms

$$
\begin{aligned}
& \int_{0}^{1} \int_{\mathbb{R}}\left(2\left|\mathcal{L}\left\{u_{k}\right\}\right|^{2}\left|\mathcal{L}\left\{w_{k}\right\}\right|^{2}+2\left|\mathcal{L}\left\{u_{k}\right\}\right|^{2} \mathcal{L}\left\{u_{k}\right\} \mathcal{L}\left\{\bar{w}_{k}\right\}\right. \\
& \left.+\left(\mathcal{L}\left\{u_{k}\right\}\right)^{2}\left(\mathcal{L}\left\{\bar{w}_{k}\right\}\right)^{2}+2\left|\mathcal{L}\left\{w_{k}\right\}\right|^{2} \mathcal{L}\left\{u_{k}\right\} \mathcal{L}\left\{\bar{w}_{k}\right\}\right) d t d z
\end{aligned}
$$

are estimated using the boundedness of $H^{1}(\mathbb{R})$ solutions of linear Schrödinger equations in $L^{\infty}(\mathbb{R})$, and the following lemma, which is a straightforward consequence of the localization lemma :

In the notation of the Concentration-Compactness Lemma the following estimates hold

$$
\begin{aligned}
& \int_{\left|t-t_{k}\right| \leq t_{c}}\left|\mathcal{L}\left\{w_{k}\right\}\right|^{2} d t \leq C \epsilon \\
& \int_{\left|t-t_{k}\right| \geq t_{c}}\left|\mathcal{L}\left\{u_{k}\right\}\right|^{2} d t \leq C \epsilon,
\end{aligned}
$$

where $t_{c}=\frac{t_{1}+t_{2}}{2}$.

Overall, we have

$$
\langle H\rangle\left\{v_{m_{k}}\right\}>\langle H\rangle\left\{w_{k}\right\}+\langle H\rangle\left\{u_{k}\right\}+\alpha(\epsilon)
$$

where $\alpha(\epsilon)$ is independent of $k$ and goes to 0 as $\epsilon \rightarrow 0$. Thus splitting causes $\langle H\rangle$ to violate subadditivity, a contradiction. 


\subsection{Minimization theorem}

Theorem 3.2.1 Let $\alpha_{2}>0$ and $\alpha_{3}=0$. Then there exists a solution to the following constrained minimization problem :

Minimize

$$
\langle H\rangle\{v\}=\alpha_{2} \int_{\mathbb{R}}\left|v_{t}\right|^{2} d t-\frac{1}{2} \int_{0}^{1} \int_{\mathbb{R}}\left|\mathcal{L}\left(z^{\prime}\right)\{v\}\right|^{4} d t d z^{\prime}
$$

over the set of admissible functions

$$
A_{\lambda}=\left\{v \in H^{1}(\mathbb{R}), \int_{\mathbb{R}}|v|^{2}=\lambda\right\}
$$

Moreover, every minimizing sequence has a subsequence which converges strongly in $H^{1}(\mathbb{R})$.

Remark: We note that the constraint $\int_{\mathbb{R}}|v|^{2}=\lambda$ is quite natural, as the $L^{2}$ norm of the initial data is preserved by solutions of the Euler-Lagrange equation (7). Posing the problem in this way is also critical to proof of the stability of the ground state which is given in a later section.

Proof:

We follow the arguments of $[6,38]$. The idea is to first show strong convergence in $L^{2}(\mathbb{R})$ by using Lions' concentration-compactness principle. This involves using structural properties of the Hamiltonian to rule out possible loss of compactness. Strong convergence in $L^{2}(\mathbb{R})$, along with an appropriate Sobolev inequality, implies convergence of the quartic term in the Hamiltonian. These results, in combination with lower semicontinuity of the $H^{1}(\mathbb{R})$ norm, give the existence of a minimizer. We show a posteriori that all minimizing sequences have a subsequence which converges strongly in $H^{1}(\mathbb{R})$.

We first argue that $I_{\lambda}>-\infty$. To prove the lower bound, we use the Sobolev inequality [4]

$$
\|\mathcal{L} v\|_{L^{4}}^{4} \leq C|| \mathcal{L} v_{t}\left\|_{L^{2}}\right\| \mathcal{L} v\left\|_{L^{2}}^{3}=C\right\| v_{t}\left\|_{L^{2}}\right\| v\left\|_{L^{2}}^{3}=C \lambda^{3 / 2}\right\| v_{t} \|_{L^{2}} .
$$

Integrating the inequality over $z^{\prime}$ gives

$$
\int_{0}^{1} \int_{-\infty}^{+\infty}|\mathcal{L}(z) v|^{4} d t d z \leq C \lambda^{3 / 2}\left\|v_{t}\right\|_{L^{2}}
$$

Thus

$$
\langle H\rangle(v) \geq\left\|v_{t}\right\|_{L^{2}}^{2}-C \lambda^{3 / 2}\left\|v_{t}\right\|_{L^{2}}=\left(\left\|v_{t}\right\|_{L^{2}}^{2}-\frac{C \lambda^{3 / 2}}{2}\right)^{2}-\frac{C^{2} \lambda^{3}}{4}>-\infty,
$$

for all $v \in H^{1}(\mathbb{R})$. Taking the infimum over $v \in A_{\lambda}$ gives the desired result.

Let $v_{k}$ be a minimizing sequence for $\langle H\rangle(v)$. By the previous inequality, 
$\left\|v_{k}\right\|_{H^{1}}$ must be bounded. By Alaoglu's theorem, there exists a weakly converging subsequence in $H^{1}(\mathbb{R}), v_{k_{m}}$. We will prove strong convergence of $v_{k_{m}}$ to a minimizer in $H^{1}(\mathbb{R})$, and first establish strong convergence in $L^{2}(\mathbb{R})$.

From previous analysis, we conclude that the minimizing sequence remains localized as $m \rightarrow \infty$. That is, for any $\epsilon>0$ there exists an $R>0$ such that

$$
\int_{-R}^{+R}\left|w_{m}(t)\right|^{2} d t>\lambda-\epsilon
$$

where $w_{m}(t)=v_{k_{m}}\left(t-t_{m}\right)$. Now $w_{m} \rightarrow w^{*}$ for some $w^{*} \in H^{1}(\mathbb{R})$. For any $R>0$, the embedding $H^{1}(\mathbb{R}) \hookrightarrow L^{2}([-R, R])$ is compact and we have

$$
\int_{-R}^{R}\left|w^{*}\right|^{2} d t=\lim _{m \rightarrow \infty} \int_{-R}^{+R}\left|w_{m}\right|^{2} d t .
$$

Together with (25), this implies

$$
\int_{-\infty}^{+\infty}\left|w^{*}\right|^{2} d t>\lambda-\epsilon \text { for any } \epsilon>0
$$

and therefore

$$
\int_{-\infty}^{+\infty}\left|w^{*}\right|^{2} d t=\lambda
$$

This norm convergence, along with weak convergence in $L^{2}(\mathbb{R})$, gives strong convergence in $L^{2}(\mathbb{R})$.

Since $w_{m}$ converges weakly to $w^{*}$ and the Sobolev norm $\|*\|_{H^{1}(\mathbb{R})}$ is weakly lower semi-continuous, we have

$$
\left\|w^{*}\right\|_{H^{1}(\mathbb{R})} \leq \liminf _{m \rightarrow \infty}\left\|w_{m}\right\|_{H^{1}(\mathbb{R})},
$$

which together with $w_{m} \rightarrow w^{*} \in L^{2}(\mathbb{R})$, implies that

$$
\left\|\partial_{t} w_{m}\right\|_{L^{2}(\mathbb{R})} \leq \liminf _{m \rightarrow \infty}\left\|\partial_{t} w_{m}\right\|_{L^{2}(\mathbb{R})}
$$

Now for any $u^{*}, u_{m} \in H^{1}(\mathbb{R})$ the Sobolev inequality gives

$$
\begin{array}{r}
\int_{-\infty}^{+\infty}\left|u_{m}-u^{*}\right|^{4} d t \leq C \int_{-\infty}^{+\infty}\left|\partial_{t} u_{m}-\partial_{t} u^{*}\right|^{2} d t\left(\int_{-\infty}^{+\infty}\left|u_{m}-u^{*}\right|^{2} d t\right)^{3 / 2} \\
\leq C\left(\int_{-\infty}^{+\infty}\left|u_{m}-u^{*}\right|^{2} d t\right)^{3 / 2}
\end{array}
$$

It follows that if $u_{m} \rightarrow u^{*}$ in $L^{2}(\mathbb{R})$,

$$
\int_{-\infty}^{+\infty}\left|u_{m}-u^{*}\right|^{4} d t \rightarrow 0
$$


Applying the same argument to $\mathcal{L}(z) w_{m}$ and $\mathcal{L}(z) w^{*}$, we establish that

$$
\mathcal{L}(z) w_{m} \rightarrow \mathcal{L}(z) w^{*} \text { in } L^{4}(\mathbb{R}) .
$$

and so

$$
\left\|\mathcal{L}(z) w^{*}\right\|_{L^{4}(\mathbb{R})}=\lim _{m \rightarrow \infty}\left\|\mathcal{L}(z) w_{m}\right\|_{L^{4}(\mathbb{R}) .}
$$

Combing (26) and (27),

$$
\langle H\rangle\left(w^{*}\right) \leq \liminf _{m \rightarrow \infty}\langle H\rangle\left(w_{m}\right),
$$

which can only happen if

$$
\langle H\rangle\left(w^{*}\right)=\lim _{m \rightarrow \infty}\langle H\rangle\left(w_{m}\right),
$$

so the weak limit $w^{*}$ is a minimizer. Furthermore, by (28)

$$
\left\|\partial_{t} w^{*}\right\|_{L^{2}(\mathbb{R})}=\lim _{m \rightarrow \infty}\left\|\partial_{t} w_{m}\right\|_{L^{2}(\mathbb{R}) .}
$$

Together with weak convergence, this implies strong convergence of $\partial_{t} w_{m}$ in $L^{2}(\mathbb{R})$, so $w_{m} \rightarrow w^{*}$ strongly in $H^{1}(\mathbb{R})$

\section{Properties of the ground state}

\subsection{Regularity}

The minimizer for the constrained minimization problem is also a weak solution to the Euler-Lagrange equation

$$
-\omega v+\alpha_{2} v_{t t}+\langle C\rangle\{v\}=0
$$

If we rewrite (29) in the form

$$
v_{t t}=\frac{1}{\alpha_{2}}(\omega v-\langle C\rangle\{v\})=f(v),
$$

where $f(v) \in H^{1}(\mathbb{R})$ by continuity of $\langle C\rangle\{v\}$, we may use standard elliptic regularity theory [15] to conclude that $v \in H^{3}(\mathbb{R})$. Again, by continuity of $\langle C\rangle\{v\}$,

$$
\omega v-\alpha_{2} v_{t t}=\langle C\rangle\{v\} \in H^{3}(\mathbb{R}),
$$

forcing $v \in H^{5}(\mathbb{R})$. We repeat this procedure indefinitely, obtaining $v \in H^{s}(\mathbb{R})$ for every $s \geq 1$, or $v \in C^{\infty}(\mathbb{R})$. We note however that the $H^{s}(\mathbb{R})$ norm of $v$ may depend on $\alpha_{2}$. 


\subsection{Stability}

It is clear that the minimizer is not unique, as any translation $v\left(\cdot+\tau_{0}\right), \tau_{0} \in \mathbb{R}$, or rotation $e^{i \theta} v, \theta \in \mathbb{R}$, of the minimizer is also a solution of the constrained minimization problem. Also, it is not known that translations and rotations give all possible minimizers. From now on we consider the class of ground state solutions $\mathcal{S}_{\lambda}=\left\{v_{g} \in A_{\lambda},\langle H\rangle\left(v_{g}\right)=I_{\lambda}\right\}$. Using the strong convergence of minimizing sequences and conservation laws for $(7)$, one can show that the minimizer is stable in the following orbital sense.

Theorem 4.2.1 Let $\mathcal{S}_{\lambda}$ be the set of ground states $\mathcal{S}_{\lambda}=\left\{v_{g} \in A_{\lambda},\langle H\rangle\left(v_{g}\right)=\right.$ $\left.I_{\lambda}\right\}$. For any $\epsilon>0$, there exists a $\delta>0$ such that if $\inf _{\mathcal{S}_{\lambda}}\left\|v-v_{g}\right\|_{H^{1}} \leq \delta$, then the solutions of (7) corresponding to initial data $v$ and $v_{g}$, denoted $v(z)$ and $v_{g}(z)$, satisfy $\sup _{z} \inf _{\mathcal{S}_{\lambda}}\left\|v(z)-v_{g}(z)\right\|_{H^{1}} \leq \epsilon$.

Proof:

We argue by contradiction. Let $v_{k}(0)$ be a sequence of initial conditions such that inf $\mathcal{S}_{\lambda}\left\|v_{k}(0)-v_{g}\right\|_{H^{1}} \rightarrow 0$, and assume that $v_{k}(z)$ and $v_{g}(z)$ satisfy $\sup _{z} \inf _{\mathcal{S}_{\lambda}}\left\|v_{k}(z)-v_{g}(z)\right\|_{H^{1}} \geq \epsilon$ for some $\epsilon>0$. For definiteness, let $z_{n}$ to be the first time that $\inf _{\mathcal{S}_{\lambda}}\left\|v_{k}(z)-v_{g}(z)\right\|_{H^{1}}=\epsilon$. By conservation of the $L^{2}$ norm and of the Hamiltonian, we have

$$
\begin{aligned}
\int_{\mathbb{R}}\left|v_{k}\left(z_{n}\right)\right|^{2} d t & =\int_{\mathbb{R}}\left|v_{k}(0)\right|^{2} d t \\
\langle H\rangle\left\{v_{k}\left(z_{n}\right)\right\} & =\langle H\rangle\left\{v_{k}(0)\right\} .
\end{aligned}
$$

By the assumption on $v_{k}(0)$ and continuity of $\langle H\rangle$, we have

$$
\begin{array}{r}
\int_{\mathbb{R}}\left|v_{k}\left(z_{n}\right)\right|^{2} d t=\int_{\mathbb{R}}\left|v_{k}(0)\right|^{2} d t \rightarrow \lambda \\
\langle H\rangle\left\{v_{k}\left(z_{n}\right)\right\}=\langle H\rangle\left\{v_{k}(0)\right\} \rightarrow\langle H\rangle\left\{v_{g}\right\} .
\end{array}
$$

By choosing for example $w_{k}=\frac{\lambda^{\frac{1}{2}} v_{k}\left(z_{n}\right)}{\left(\int_{\mathbb{R}}\left|v_{k}\left(z_{n}\right)\right|^{2} d t\right)^{\frac{1}{2}}}$, let $w_{k}$ be a sequence of $H^{1}$ functions such that

$$
\left\|w_{k}-v_{k}\left(z_{n}\right)\right\|_{H^{1}} \rightarrow 0
$$

and $\int_{\mathbb{R}}\left|w_{k}(z)\right|^{2} d t=\lambda$. By continuity of $\langle H\rangle, w_{k}$ is a minimizing sequence and must have a subsequence $w_{m_{k}}$ which converges to a ground state. But

$$
\left\|v_{k}\left(z_{n}\right)-v_{g}(z)\right\|_{H^{1}} \leq\left\|v_{k}\left(z_{n}\right)-w_{m_{k}}\right\|_{H^{1}}+\left\|w_{m_{k}}-v_{g}(z)\right\|_{H^{1}},
$$

and taking the infimum over $\mathcal{S}_{\lambda}$ gives

$\epsilon=\inf _{\mathcal{S}_{\lambda}}\left\|v_{k}\left(z_{n}\right)-v_{g}(z)\right\|_{H^{1}} \leq\left\|v_{k}\left(z_{n}\right)-w_{m_{k}}\right\|_{H^{1}}+\inf _{\mathcal{S}_{\lambda}}\left\|w_{m_{k}}-v_{g}(z)\right\|_{H^{1}} \rightarrow 0$,

a contradiction. Thus the class of ground states must be orbitally stable. 


\section{$5 \quad$ Numerical studies}

\subsection{Solution of the eigenvalue problem}

In Fourier space, the Euler-Lagrange equation (29) becomes

$$
-\omega \hat{v}-\alpha_{2} k^{2} \hat{v}+\langle\hat{C}\rangle\{v\}=0 .
$$

We propose the following explicit iteration scheme

$$
-\omega_{n} \hat{v}_{n+1}-\alpha_{2} k^{2} \hat{v}_{n+1}+\langle\hat{C}\rangle\left\{v_{n}\right\}=0,
$$

so that

$$
\hat{v}_{n+1}=\frac{\langle\hat{C}\rangle\left\{v_{n}\right\}}{\omega_{n}+\alpha_{2} k^{2}}
$$

If we multiply (30) by $\overline{\hat{v}}(k)$ and integrate over $k$, we can derive a formula for $\omega_{n+1}$.

$$
\omega_{n+1}=\frac{\int_{\mathbb{R}} \overline{\hat{v}}_{n+1}\langle\hat{C}\rangle\left\{v_{n+1}\right\} d k-\alpha_{2} \int_{\mathbb{R}} k^{2}\left|\hat{v}_{n+1}\right|^{2} d k}{\int_{\mathbb{R}}\left|\hat{v}_{n+1}\right|^{2} d k} .
$$

This idea also suggests a definition for a relaxation factor to hasten convergence $[21,31]$,

$$
s_{n+1}=\frac{\omega_{n+1} \int_{\mathbb{R}}\left|\hat{v}_{n+1}\right|^{2} d k}{\int_{\mathbb{R}} \overline{\hat{v}}_{n+1}\langle\hat{C}\rangle\left\{v_{n+1}\right\} d k-\alpha_{2} \int_{\mathbb{R}} k^{2}\left|\hat{v}_{n+1}\right|^{2} d k} .
$$

The factor $s_{n}$ can be used to compensate the nonlinearity, and as the scheme converges, $s_{n} \rightarrow 1$. We also note that convergence depends strongly on the initial guess, which is typically taken to be Gaussian. Incorporating the relaxation factor, the overall scheme becomes

$$
\begin{gathered}
\hat{v}_{n+1}=\left(s_{n}\right)^{p} \frac{\langle\hat{C}\rangle\left\{v_{n}\right\}}{\omega_{n}+\alpha_{2} k^{2}}, \\
\omega_{n+1}=\frac{\int_{\mathbb{R}} \overline{\hat{v}}_{n+1}\langle\hat{C}\rangle\left\{v_{n+1}\right\} d k-\alpha_{2} \int_{\mathbb{R}} k^{2}\left|\hat{v}_{n+1}\right|^{2} d k}{\int_{\mathbb{R}}\left|\hat{v}_{n+1}\right|^{2} d k}, \\
s_{n+1}=\frac{\omega_{n+1} \int_{\mathbb{R}}\left|\hat{v}_{n+1}\right|^{2} d k}{\int_{\mathbb{R}} \overline{\hat{v}}_{n+1}\langle\hat{C}\rangle\left\{v_{n+1}\right\} d k-\alpha_{2} \int_{\mathbb{R}} k^{2}\left|\hat{v}_{n+1}\right|^{2} d k},
\end{gathered}
$$

where we use $p=1.5$.

To confine our search for minimizers to a fixed level set of $L^{2}(\mathbb{R})$, we normalize the result of each iteration so that its energy is that of the initial guess,

$$
\left\|v_{n}\right\|_{L^{2}}^{2}(\mathbb{R})=\left\|v_{0}\right\|_{L^{2}}^{2}(\mathbb{R})=\lambda .
$$

In practice the algorithm is 
1. Choose an initial profile $\hat{v}_{0}$, typically Gaussian.

2. Compute $\omega_{0}$ with $\hat{v}_{0}$.

3. Compute $s_{0}$ with $\hat{v}_{0}$ and $\omega_{0}$.

4. Compute $\hat{v}_{1}$ using the scheme.

5. Rescale $\hat{v}_{1}$ so that $\left\|\hat{v}_{1}\right\|_{L^{2}}^{2}(\mathbb{R})=\left\|\hat{v}_{0}\right\|_{L^{2}}^{2}(\mathbb{R})$.

6. Compute $\omega_{1}$ and $s_{1}$.

7. Repeat 3,4 and 5 until desired accuracy reached.

\subsection{Solution of evolution equations}

Both the full evolution equation (2) and averaged equation (7) can easily be solved with a version of the well known Fourier split-step scheme, which applies to a wide class of NLS-type equations. Given an evolution equation of the form

$$
i u_{z}+\mathcal{L}\{u\}+\mathcal{N}\{u\}=0,
$$

where $\mathcal{L}$ is a self-adjoint operator on a Hilbert space and $\mathcal{N}$ is a continuous nonlinear operator, the solution may be written formally as

$$
u(z, t)=u(0, t) e^{i \int_{0}^{z}(\mathcal{L}(s)\{u\}+\mathcal{N}(s)\{u\}) d s} .
$$

We consider the evolution for a small propagation step $\Delta z$ so that we may approximate (31) using a formal Taylor expansion

$$
\begin{array}{r}
u(\Delta z, t)=u(0, t) e^{i \int_{0}^{\Delta z}(\mathcal{L}(s)\{u\}+\mathcal{N}(s)\{u\}) d s} \\
\approx u(0, t) e^{i \int_{0}^{\Delta z / 2} \mathcal{L}(s)\{u\}} e^{i \int_{0}^{\Delta z} \mathcal{N}(s)\{u\} d s} e^{i \int_{0}^{\Delta z / 2} \mathcal{L}(s)\{u\} d s},
\end{array}
$$

with a local error on the order of $(\Delta z)^{3}$. Performing the approximation for $\mathcal{O}\left(\frac{1}{\Delta z}\right)$ time steps gives a global error on the order of $(\Delta z)^{2}$. Formally, $e^{i \int_{0}^{z} \mathcal{L}(s)\{u\} d s}$ is the semigroup for the linear evolution

$$
i u_{z}+\mathcal{L}\{u\}=0
$$

and can be computed explicitly in Fourier domain. Also,

$$
e^{i \int_{0}^{z} \mathcal{N}(s)\{u\} d s}
$$

is the solution operator for the evolution equation

$$
i u_{z}+\mathcal{N}\{u\}=0,
$$

and can be computed either by a standard ODE method such as fourth-order Runge-Kutta or, in special cases, by using conservation laws for the equation.

The overall scheme becomes 
1. Choose an initial profile $u(0, t)$ and compute its Fourier transform with the FFT.

2. In Fourier space, evolve the linear dispersive operator for $\Delta z / 2$.

3. Evolve the nonlinear operator for $\Delta z$.

4. Evolve the linear dispersive operator for $\Delta z / 2$.

5. Repeat 3 and 4 until the final propagation step.

6. Evolve the linear dispersive operator for $\Delta z / 2$ and compute the inverse Fourier transform with the IFFT.

\subsection{Existence of nearly periodic solutions}

Combining the averaging and minimization results, we see that there exist stationary solutions for (7) that evolve nearly periodically for (2). The ground state $v_{g}(t)$ for the variational problem corresponds to a standing wave solution for $(7), v(z, t)=\exp (i \omega z) v_{g}(t)$. The averaging theorem gives that

$$
\left\|u-\mathcal{L}\left(\frac{z}{\epsilon}\right)\{v(z, t)\}\right\|_{L^{\infty}\left(\left[0, \frac{z^{*}}{\epsilon}\right], H^{s-3}(\mathbb{R})\right)} \leq \epsilon
$$

so that $u(z, t)$ is nearly periodic on the scale of validity for the averaging theorem. Moreover, by well-posedness of the averaged equation, the same result is true for initial data chosen close to the class of ground states inf $\mathcal{S}_{\lambda}\left\|v-v_{g}\right\|_{H^{1}} \leq$ $\epsilon$.

We define a higher order dispersion managed soliton to be an element from the class of ground states $\mathcal{S}_{\lambda}$ and demonstrate the existence of such solutions numerically. Figure 1 shows the shape of the ground state solution for the parameters

$$
\tilde{d}_{2}\left(z^{\prime}\right)=\tilde{d}_{3}\left(z^{\prime}\right)= \begin{cases}5.0 & \text { if } z^{\prime} \in[0, .25) \text { or } z^{\prime} \in[.75,1.0) \\ -5.0 & \text { if } z^{\prime} \in[.25, .75)\end{cases}
$$

and $\alpha_{2}=1.0, \epsilon=0.1$. The solution is computed on the time domain $[-30,30]$ with 2048 Fourier modes. The logarithm of the amplitude $|v(t)|$ is plotted vs. time on the interval $[-20,20]$. One observes a nearly Gaussian central peak, along with many secondary peaks which decay rapidly. This is similar to the structure of the ground states observed for dispersion management at second order $[1,28]$.

From the averaging theorem, one would expect the ground state to evolve nearly periodic for $z \sim \mathcal{O}(10)$. Figure 2 depicts the evolution of the maximum amplitude of the ground state for the corresponding full equation. The individual oscillations are due to the linear compensation of dispersion, and we observe that the evolution of the amplitude is, in fact, nearly periodic on $z$ scales much longer than those predicted by the averaging theorem. 


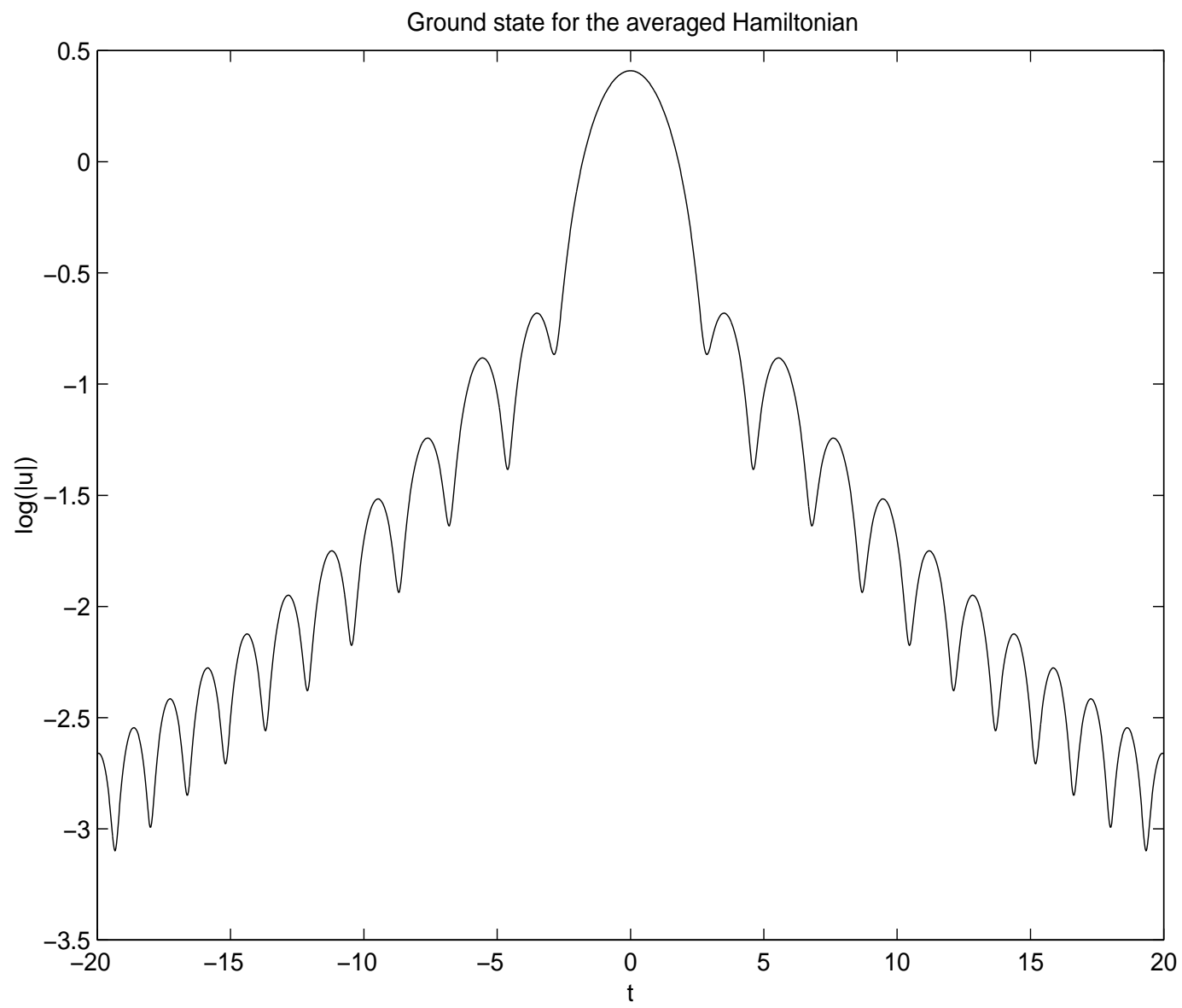

Figure 1: High order dispersion managed soliton 


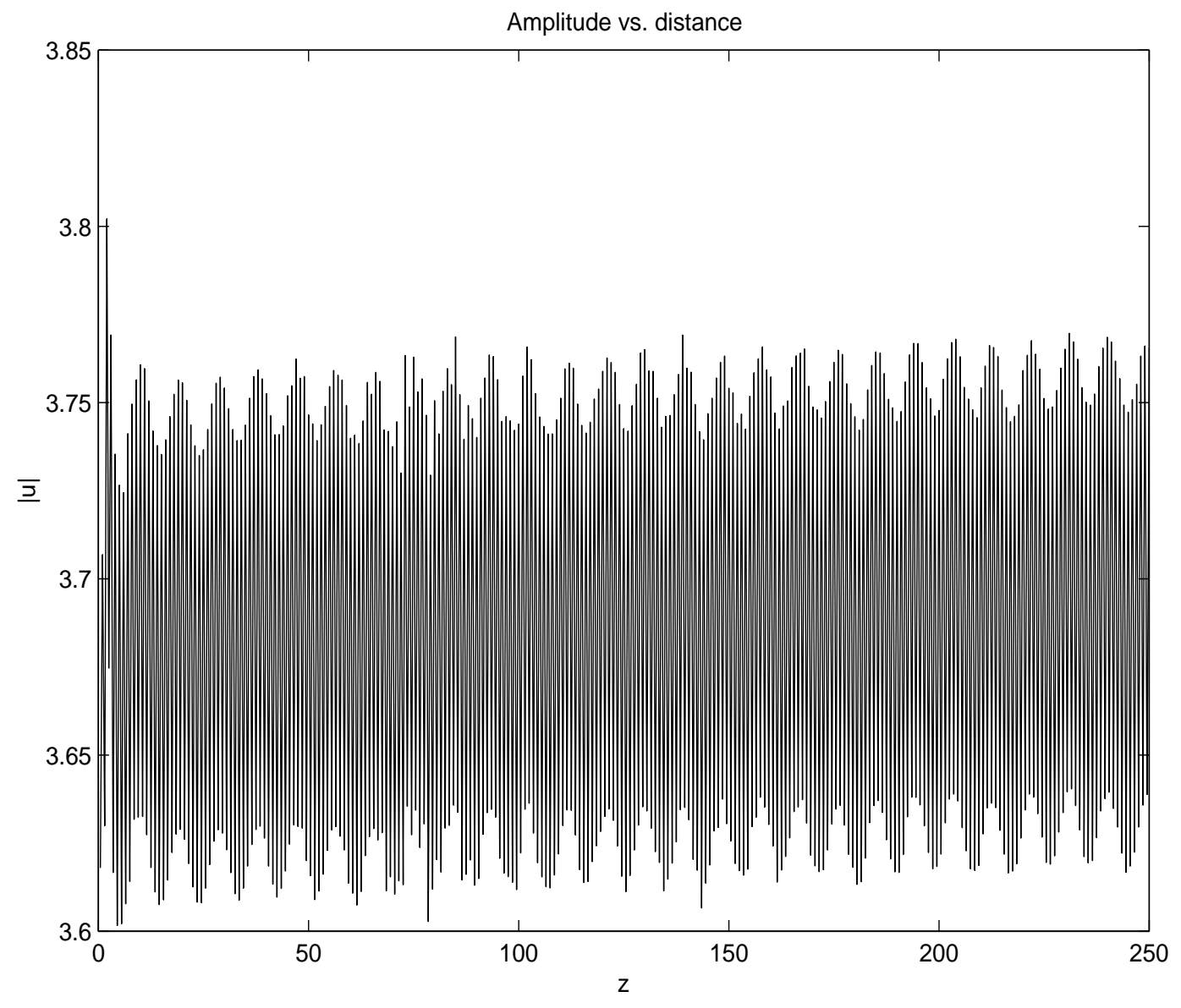

Figure 2: Nearly periodic evolution of the ground state 


\section{Acknowledgments}

We are grateful to all of the reviewers, who provided many insightful suggestions and comments. We are particularly indebted to the reviewer who provided an alternate proof of Lemma 3.1.2, which was used in this text. We also would like to thank Ildar Gabitov for bringing this problem to our attention. J. Moeser was supported by NSF under grants DMS-0073923 and DMS-9810751. C. Jones was supported by NSF under Grant No. DMS-0073923. Part of this work was done when V. Zharnitsky was visiting Program in Applied and Computational Mathematics at Princeton University. He would like to thank Ingrid Daubechies for her hospitality and for providing a stimulating research environment. V. Zharnitsky was supported by NSF under grant No. DMS-0219233. He would also like to acknowledge partial support under grant No. DMS-0073923.

\section{References}

[1] M. J. Ablowitz, G. Biondini. Multiscale pulse dynamics in communication systems with strong dispersion management. Opt. Lett. 23, p. 1668-1670, 1998.

[2] M. J. Ablowitz, T. Hirooka. Resonant nonlinear interactions in strongly dispersion-managed transmission systems. Opt. Lett. 25, p. 1750-1752, 2000.

[3] G. P. Agrawal. Fiber-Optic Communication Systems. 2nd ed., John Wiley and Sons, Inc. New York, 1997.

[4] H. Brezis. Analyse fonctionelle - Theorie et applications. Masson, New York, p. $129,1987$.

[5] S. K. Burtsev, I. Gabitov. Four-wave mixing in fiber links with dispersion management. in Proc. II Int. Symp. Phys. Applica. Opt. Solitons Fibers. Kyoto, Japan, p. 261-265, 1997.

[6] T. Cazenave. An introduction to nonlinear Schrödinger equations. UFRJ, Rio de Janeiro, p. 183-195, 1993.

[7] L. du Mouza, E. Seve, H. Mardoyn, S. Wabnitz, P. Sillard, P. Nouchi. Highorder dispersion-managed solitons for dense wavelength-division multiplexed transmissions. Opt. Lett. 26, p. 1128, 2001.

[8] F. Favre, D. Le Guen, M. L. Moulinard, M. Henry, T. Georges. 320Gbit/s soliton WDM transmission over $1300 \mathrm{~km}$ with $100 \mathrm{~km}$ dispersion compensated spans of standard fibre. Elec. Lett. 33(25), p. 2135-2136, 1997. 
[9] F. Favre, D. Le Guen, T. Georges. Experimental evidence of pseudoperiodical soliton propagation in dispersion managed link. Elec. Lett. 34(19), 1868-1869, 1998.

[10] I. Gabitov, E. G. Shapiro, S. K. Turitsyn. Asymptotic breathing pulse in optical transmission systems with dispersion compensation. Phys. Rev. E 55, p. 3624-3633, 1995.

[11] E.A. Golovchenko, V.J Mazurczyk, D.G Duff, S.M. Abbott. Four-wave mixing penalties in long-haul WDM transmission links. IEEE Photon. Technol. Lett., 11, p.821-823, 1999.

[12] M. Kunze. Bifurcation from the essential spectrum without sign condition on the nonlinearity. To appear in Proc. Roy. Soc. Edinburgh, Ser. A.

[13] M. Kunze. A variational problem with lack of compactness related to the nonlinear Schrödinger equation. Submitted 2001.

[14] T. I. Lakoba, J. Yang, D. J. Kaup, B. A. Malomed. Conditions for stationary pulse propagation in the strong dispersion management regime. Opt. Comm. 149, p. 366-375, 1998.

[15] E. H. Lieb, M. Loss. Analysis. AMS, Providence, p. 226, 1997.

[16] C. Lin, H. Kogelnik, L. G. Cohen. Optical Pulse Equalization and Low Dispersion Transmission in Single-Mode Fibers in the 1.3 - $1.7 \mathrm{~mm}$ Spectral Region. Opt. Lett. 5, p. 476-478, 1980.

[17] P. L. Lions. The concentration-compactness principle in the Calculus of Variations. The locally compact case, part 1, Ann. Inst. Henri Poincare 1(2), p. $109-145,1984$.

[18] Y. Liu. Challenging the limits of chromatic dispersion. in Lightwave, a publication of Pennwell Corporation, 2001.

[19] F. Liu, J. Bennike, S. Dey, C. Rasmussen, H. Mikkelsen, P. Mamyshev, D. Gapontse, V. Ivshin. 1.6 Tbit/s (40x42.7 Gbit/s) transmission over $3600 \mathrm{~km}$ UltraWave ${ }^{t m}$ fiber with all-Raman amplified $100 \mathrm{~km}$ terrestrial spans using ETDM transmitter and receiver. OFC 2002 Post Deadline Digest session FC7 1-3, Optical Fiber Communications, Anaheim, California, March 17-21, 2002.

[20] P. M. Lushnikov, Dispersion-managed soliton in optical fibers with zero average dispersion, Optics Letters, 25(16), p. 1144-1146, 2000.

[21] P. M. Lushnikov, Dispersion-managed soliton in a strong dispersion map limit, Optics Letters, 26(20), p. 1535-1537, 2001.

[22] P. V. Mamyshev, N. A. Mamysheva. Pulse-overlapped dispersion managed data transmission and intrachannel four-wave mixing. Opt. Lett. 24, p. 14541456, 1999. 
[23] M. Manna, E.A. Golovchenko. FWM resonances in dispersion slopematched and non-zero-dispersion fiber maps: impact on system performance. Optical Fiber Communication Conference and Exhibition. Technical Digest, Anaheim, CA, USA,17-22 March 2001.

[24] J. Moeser, I. Gabitov, C.K.R.T. Jones. Pulse stabilization by high order dispersion management, Optics Letters, Vol. 27, Issue 24, p. 2206-2208, 2002.

[25] L. F. Mollenauer, P. V. Mamyshev, J. Gripp, M. J. Neubelt, N. Mamysheva, L. Gruner-Nielsen, T. Veng. Demonstration of massive wavelength-division multiplexing over transoceanic distances by use of dispersion-managed solitons. Opt. Lett. 25(10), p. 704, 2000.

[26] M. Murakami, H. Maeda, and T. Imai, Long-Haul 16x10 Gb/s WDM Transmission Experiment Using Higher Order Fiber Dispersion Management Technique. IEEE Photonics Technology Letters, vol. 11, p. 898-900, 1999.

[27] M. Nakazawa, H. Kubota, K. Suzuki, E. Yamada. Recent progress in soliton transmission technology. Chaos 10(3), 486-514, 2000.

[28] J. H. B. Nijhof, N. J. Doran, W. Forysiak, F. M. Knox. Stable soliton-like propagation in dispersion managed systems with net anomalous, zero, and normal dispersion. Elec. Lett. 33, p. 1726-1728, 1997.

[29] J. H. B. Nijhof, N. J. Doran, W. Forysiak, A. Berntson. Energy enhancement of dispersion managed solitons and WDM. Elec. Lett. 34 p. 481-483, 1998.

[30] A. Pazy. Semigroups of Linear Operators and Applications to Partial Differential Equations. Springer-Verlag, New York, p. 190, 1983

[31] V. I. Petviashvili and O. A. Pokhotelov. Solitary Waves in Plasmas and in the Atmosphere. Gordon and Breach, Philadelphia, p. 248, 1992

[32] J. A. Sanders and F. Verhulst. Averaging Methods in Nonlinear Dynamical Systems. Springer-Verlag, New York, p. 41, 1985.

[33] I. Segal. Non-Linear Semi-Groups. The Annals of Mathematics, Second Series, Volume 78, Issue 2, p. 339-364, 1963.

[34] N. J. Smith, N. J. Doran, F. M. Knox, W. Forysiak. Energy-scaling characteristics of solitons in strongly dispersion-managed fibers. Opt. Lett. 21(24), p. 1981-1983, 1996.

[35] W. A. Strauss. Nonlinear Wave Equations. AMS, Providence, p. 18, 1989.

[36] M. Suzuki, I. Morita, N. Edagawa, S. Yamamoto, H. Taga, S. Akiba. Reduction of Gordon-Haus timing jitter by periodic dispersion compensation in soliton transmission. Elec. Lett. 31, p. 2027-2030, 1995. 
[37] S.K. Turitsyn, I. Gabitov, E. W. Laedke, V. K. Mezentsev, S. L. Musher, E. G. Shapiro, T. Schäfer, K. H. Spatschek. Variational approach to optical pulse propagation in dispersion compensated transmission systems, Opt. Comm. 151, p. 117-135, 1998.

[38] V. Zharnitsky, E. Grenier, C. K. R. T Jones, S. K. Turitsyn. Stabilizing effects of dispersion management. Physica D 152-153, p. 794-817, 2001. 